\title{
LATTICE BOLTZMANN FORMULATION FOR LINEAR VISCOELASTIC FLUIDS USING AN ABSTRACT SECOND STRESS
}

PAUL J. DELLAR*

\begin{abstract}
The kinetic theory of gases implies an independent evolution equation for the momentum flux tensor that closely resembles an evolution equation for the elastic stress in continuum descriptions of viscoelastic liquids. However, kinetic theory leads to a non-objective convected derivative for the evolution of the deviatoric stress, and a fixed relation between the stress relaxation rate and the viscosity. We show that simulations of freely decaying shear flow using the standard two-dimensional lattice Boltzmann kinetic model develop a tangential stress consistent with this non-objective convected derivative, and this fixed relation between parameters. By contrast, viscoelastic liquids are typically modelled by an upper convected derivative, and with two independent parameters for the viscosity and stress relaxation rate. Although we are unable to obtain an upper convected derivative from kinetic theory with a scalar distribution function, we show that introducing a general linear coupling to a second stress tensor yields the linear Jeffreys viscoelastic model with three independent parameters in the incompressible limit. Unlike previous work, we do not attempt to represent the additional stress through moments of additional distribution functions, but treat it only as an abstract tensor that couples to the corresponding tensorial moment of the hydrodynamic distribution functions. This greatly simplifies the derivation, and the implementation of flows driven by body forces. The utility of the approach is demonstrated through simulations of Stokes' second problem for an oscillating boundary, of the four-roller mill, and of three-dimensional Arnold-Beltrami-Childress and Taylor-Green flows.
\end{abstract}

Submitted 7 October 2013, revised 20 May 2014, accepted 24 June 2014 by SIAM Journal on Scientific Computing

1. Introduction. Lattice Boltzmann algorithms have achieved notable successes for simulating simple Newtonian fluids, multiphase flows, suspensions with resolved microstructure, and macroscopic continuum models for liquid crystals, electrically conducting fluids, and strongly magnetised plasmas with anisotropic stress-strain relations [58, 25, 12, 2, 18, 20, 22]. Lattice Boltzmann approaches for continuum models of viscoelastic liquids are notably less advanced. This is perhaps surprising given the many similarities between such models and kinetic theory. One of the simplest models for viscoelastic liquids is the linear Maxwell model $[8,51,71,76]$

$$
\mathrm{T}+\tau \partial_{t} \mathrm{~T}=\mu \mathrm{E}
$$

Maxwell originally proposed this model for rarefied gases [65]. It generalises the usual instantaneous relation $\mathrm{T}=\mu \mathrm{E}$ between the deviatoric stress $T$ and the strain rate $E$ in a Newtonian fluid with viscosity $\mu$ by allowing the stress to relax over a timescale $\tau$ set by the the frequency of collisions between particles. The instantaneous relation is recovered for solutions of (1.1) that vary slowly on timescales much longer than $\tau$. Seeking slowly varying solutions is the key ingredient of the Chapman-Enskog perturbation expansion that derives the Navier-Stokes equations from kinetic theory [13, 15]. The separate evolution equation for $\mathrm{T}$ is what distinguishes genuinely non-Newtonian or viscoelastic liquids from generalised Newtonian fluids. The stress in the latter is a function of the local, instantaneous strain rate, typically of the form $\mathrm{T}=\mu(\|\mathrm{E}\|) \mathrm{E}$, with $\|\mathrm{E}\|=(\mathrm{E}: \mathrm{E})^{1 / 2}$. There is a well-established lattice Boltzmann approach for simulating such fluids, and for the mathematically identical Smagorinsky turbulence model [78, 1, 79, 45, 95, 82, 72, 22].

Heuristic models for rarefied gases such as (1.1) were superceded by the Boltzmann equation that gives a complete description of a dilute monatomic gas. It leads (see section 2) to the nonlinear evolution equation

$$
\mathrm{T}+\tau\left[\partial_{t} \mathrm{~T}+\mathbf{u} \cdot \nabla \mathrm{T}+\mathrm{T} \cdot \nabla \mathbf{u}+(\nabla \mathbf{u})^{\mathrm{T}} \cdot \mathrm{T}\right]=\tau \rho \theta \mathrm{E}
$$

in the incompressible limit. We write the velocity gradient as $[\nabla \mathbf{u}]_{i j}=\partial_{i} u_{j}$ in suffix notation, so $[\mathrm{T} \cdot \nabla \mathbf{u}]_{i j}=T_{i k}\left(\partial_{k} u_{j}\right)$, and a superscript ${ }^{\top}$ denotes a matrix transpose. The first difficulty lies in the fixed relation $\mu=\tau \rho \theta$ between the viscosity $\mu$ and relaxation time $\tau$ for a fluid with density $\rho$ and temperature $\theta$. A satisfactory model for viscoelastic liquids requires two independent parameters for $\tau$ and $\mu$. Secondly, the partial time derivative $\partial_{t} \top$ in (1.1) has been replaced by a combination of terms $\left[\partial_{t} \boldsymbol{\top}+\cdots\right]$ involving the fluid velocity $\mathbf{u}$ and its gradient $\nabla \mathbf{u}$. The first two terms $\partial_{t} \boldsymbol{\top}+\mathbf{u} \cdot \nabla \mathrm{T}$ make up the standard material derivative for a scalar quantity, and the extra $\mathrm{T} \cdot \nabla \mathbf{u}+(\nabla \mathbf{u})^{\mathrm{T}} \cdot \mathrm{T}$ terms appear because $\mathrm{T}$ is a tensor. However, neither the partial time derivative $\partial_{t} \mathrm{~T}$ in (1.1) nor the combination $\left[\partial_{t} \mathrm{~T}+\cdots\right]$ in (1.2) transforms as required under rotations. In both models, the stress in a deforming fluid subject to an additional rigid body rotation differs from the rotation of the stress in a fluid undergoing the same deformation without rotation. The two models are thus not objective [8].

By contrast, the rheology of polymers is commonly described using the upper convected Maxwell model $[8,51,71,76]$

$$
\mathrm{T}+\tau\left[\partial_{t} \mathrm{~T}+\mathbf{u} \cdot \nabla \mathrm{T}-\mathrm{T} \cdot \nabla \mathbf{u}-(\nabla \mathbf{u})^{\mathrm{T}} \cdot \mathrm{T}\right]=\mu \mathrm{E}
$$

This model is objective, because the third and fourth terms in the material time derivative $\left[\partial_{t} \top+\cdots\right]$ have the opposite signs to those in (1.2). The lower convected Maxwell model

$$
\mathrm{T}+\tau\left[\partial_{t} \mathrm{~T}+\mathbf{u} \cdot \nabla \mathrm{T}+(\nabla \mathbf{u}) \cdot \mathrm{T}+\mathrm{T} \cdot(\nabla \mathbf{u})^{\mathrm{T}}\right]=\mu \mathrm{E}
$$

is also objective, since the tensor contractions are between $\mathrm{T}$ and the $\mathbf{u}$ rather than the $\nabla$ component of the dyad $\nabla \mathbf{u}$, and has been used to model suspensions of discoid particles. Linear combinations of (1.3) and (1.4) are also objective, such as the Jaumann or corotational time derivative.

The upper convected Maxwell model (1.3) may be derived from the Fokker-Planck equation for a dilute suspension of microscopic dumbbells, each comprising a pair of Brownian beads separated by a linear spring [64, 73, 9, 76]. Assuming for

\footnotetext{
*OCIAM, Mathematical Institute, Radcliffe Observatory Quarter, Oxford OX2 6GG, United Kingdom (dellar@maths.ox.ac.uk)
} 
the moment that each bead moves with the local fluid velocity, the separation vector between each pair of beads becomes a material line element $\ell$ that evolves according to

$$
\partial_{t} \ell+\mathbf{u} \cdot \nabla \ell-\ell \cdot \nabla \mathbf{u}=0
$$

in an incompressible fluid [4]. The last term represents the stretching of material elements by velocity gradients, being the velocity difference $\mathbf{u}(\mathbf{x}+\ell)-\mathbf{u}(\mathbf{x})$ between the two ends of an element. The dyad $\ell \ell$ then evolves according to the upper convected derivative. The upper convected Maxwell model (1.3) describes an elastric stress proportional to an ensemble average $\langle\ell \ell\rangle$ over many dumbbells. The additional terms $\mathrm{T}$ and $\mu \mathrm{E}$ in (1.3) arise from the beads slipping relative to their surrounding fluid, and from stochastic Brownian forces exerted on the beads by solvent molecules. Equation (1.5) also describes the advection and stretching of the magnetic field vector $\mathbf{B}$ in ideal magnetohydrodynamics, for which the magnetic Maxwell stress tensor $\mathbf{B B}-\frac{1}{2}|\mathbf{B}|^{2}$ I evolves according to the upper convected derivative.

Conversely, suppose $\phi$ is an advected scalar field that obeys $\partial_{t} \phi+\mathbf{u} \cdot \nabla \phi=0$. Its gradient $\nabla \phi$ evolves according to

$$
\partial_{t} \nabla \phi+(\mathbf{u} \cdot \nabla) \nabla \phi+(\nabla \mathbf{u}) \cdot \nabla \phi=0 .
$$

The last term has a different sign to that in (1.5), and the contraction is with the $\mathbf{u}$ rather than the $\nabla$ component of the dyad $\nabla \mathbf{u}$. These differences together ensure that $\ell \cdot \nabla \phi$ evolves as an advected scalar field, while the dyad $(\nabla \mathbf{u})(\nabla \mathbf{u})$ evolves as a lower convected tensor field. The names "upper convected" and "lower convected" arise from the use of upper indices such as $\ell^{i}$ for vector components, and lower indices such as $\partial_{i} \phi$ for components of co-vectors or 1-forms [67, 51].

Given the incompatibility between these objective nonlinear rheological models and the stress evolution equation (1.2) obtained from the Boltzmann equation, previous lattice Boltzmann approaches have targetted the linear Jeffreys model [50, 8, $51,71,76]$. This model generalises the linear Maxwell model by including the time derivative of $E$ with an additional time constant $\Lambda$,

$$
\mathrm{T}+\lambda \partial_{t} \mathrm{~T}=\mu\left(\mathrm{E}+\Lambda \partial_{t} \mathrm{E}\right)
$$

We now use $\lambda$ for the time constant for T. We reserve $\tau$ for the stress relaxation time that appears in the moment (2.4) of the kinetic equation (2.1) below, and in the Newtonian viscosity $\mu=\tau \rho \theta$. The Jeffreys model arises naturally for polymer solutions if one decomposes the total stress $\mathrm{T}=\mu^{\prime} \mathrm{E}+\widetilde{\mathrm{T}}$ into a Newtonian viscous stress $\mu^{\prime} \mathrm{E}$ due to the solvent, and an additional stress $\widetilde{\mathrm{T}}$ due to the polymers that is governed by the linear Maxwell model. It was later derived from a microscopic description of a suspension of elastic particles in a viscous fluid [33]. This decomposition of T establishes the relation $\Lambda=\lambda \mu^{\prime} /\left(\mu+\mu^{\prime}\right)$ and implies $\Lambda<\lambda$. A real polymeric liquid has a whole spectrum $\lambda_{1}, \lambda_{2}, \ldots$ of stress relaxation times. The single $\lambda$ in the Jeffreys model is identified with the longest of these, while the others are all supposed short enough to be modelled collectively by the Newtonian viscous stress. Replacing the partial time derivatives in (1.7) with upper convected derivatives leads to the popular Oldroyd-B model [67]. This model offers a good description of the Boger fluids that possess elastic properties but no significant shear-dependence of their viscosities [11, 14, 49].

Lattice Boltzmann algorithms represent the hydrodynamic variables such as density and velocity as moments of a finite set of distribution functions $f_{\alpha}$, each moving with a fixed velocity $\boldsymbol{\xi}_{\alpha}$, as described in Sec. 2. Following an earlier two-dimensional lattice gas model [24], Giraud et al. [36, 37] developed a two-dimensional lattice Boltzmann formulation for the Jeffreys model by adding two more distribution functions $f_{9}$ and $f_{10}$ with zero velocity, $\boldsymbol{\xi}_{9}=\boldsymbol{\xi}_{10}=0$. They used these additional degrees of freedom to build a second traceless stress tensor, which they coupled to the existing stress through the collision operator. This coupling enables $\mu$ and $\tau$ to be adjusted independently. The approach was later extended to three dimensions [59].

Ispolatov \& Grant [48] subsequently implemented a linear Maxwell model using an ordinary differential equation (ODE) to evolve the divergence of the elastic stress at each lattice point. Their ODE contains a forcing term $\nabla \cdot E=\nabla^{2} \mathbf{u}$ calculated using a finite difference approximation, and they included the divergence of the elastic stress as a body force in their momentum equation. This approach was pursued by Li \& Fang [62] and Frantziskonis [31], and extended to include a finite spectrum of relaxation times (typically 6 ) by Frank \& $\mathrm{Li}[29,30]$. This latter work puts the elastic stress into the second moment $\boldsymbol{\Pi}^{(0)}$ of the equilibrium distributions, following the approach used to include the Maxwell stress in lattice Boltzmann magnetohydrodynamics [18], instead of including the stress divergence as a body force. Tsutahara et al. [90] proposed a modified discrete Boltzmann equation that allows an independent adjustment of $\tau$ and $\mu$ without introducing additional degrees of freedom (see the appendix) but their equation cannot be implemented using the standard lattice Boltzmann space/time discretisation. Moving beyond linear viscoelastic theory, Onishi et al. [68, 69] simulated a population of microscopic dumbbells in a viscous fluid, whose macroscopic behavior reproduces the Oldroyd-B model. Karra [54] coupled a finite difference discretisation of the Oldroyd-B elastic stress evolution equation with a standard lattice Boltzmann hydrodynamic algorithm, while Malaspinas et al. [63] used a lattice Boltzmann advection/diffusion algorithm for each component of the elastic stress tensor. Phillips \& Roberts [72] have reviewed these different approaches, concentrating mainly on generalised Newtonian fluids.

In this paper we present a greatly simplified lattice Boltzmann formulation for the Jeffreys model using a matrix collision operator defined purely in terms of moments to couple the existing stress $T$ with a second stress $M$ local to each lattice point. Unlike previous work, we make no attempt to represent $M$ as the second moment of a set of distribution functions. Instead, we simply evolve the components of $M$ directly at each lattice point. A general linear coupling between $T$ and $M$ contains three coefficients, the overall magnitude of $\mathrm{M}$ relative to $\mathrm{T}$ being arbitrary. This set of three coefficients is in a one-to-one relation with the set of three coefficients $\mu, \lambda, \Lambda$ appearing in the Jeffreys model. Although we begin with a nonlinear and nonobjective convected derivative for $\mathrm{T}$ in (1.2), we obtain the linear Jeffreys model in the low Mach number limit relevant for simulating incompressible flow. 
2. Stress evolution in kinetic theory. We consider a finite set of distribution functions $f_{\alpha}(\mathbf{x}, t)$ for $\alpha=0,1, \ldots, N$ that evolve according to the discrete Boltzmann equation

$$
\partial_{t} f_{\alpha}+\boldsymbol{\xi}_{\alpha} \cdot \nabla f_{\alpha}=-\sum_{\beta=0}^{N} \Omega_{\alpha \beta}\left(f_{\beta}-f_{\beta}^{(0)}\right) .
$$

Each $f_{\alpha}$ propagates with constant velocity $\boldsymbol{\xi}_{\alpha}$, and interacts with the other distribution functions through the collision term on the right hand side. We use Greek indices to label discrete velocities, and reserve Roman indices for Cartesian tensor components. Hydrodynamic quantities, the density $\rho$, velocity $\mathbf{u}$, and momentum flux $\boldsymbol{\Pi}$, are defined as moments of the $f_{\alpha}$,

$$
\rho=\sum_{\alpha=0}^{N} f_{\alpha}, \quad \rho \mathbf{u}=\sum_{\alpha=0}^{N} \boldsymbol{\xi}_{\alpha} f_{\alpha}, \quad \boldsymbol{\Pi}=\sum_{\alpha=0}^{N} \boldsymbol{\xi}_{\alpha} \boldsymbol{\xi}_{\alpha} f_{\alpha}, \quad \mathcal{Q}=\sum_{\alpha=0}^{N} \boldsymbol{\xi}_{\alpha} \boldsymbol{\xi}_{\alpha} \boldsymbol{\xi}_{\alpha} f_{\alpha}
$$

The velocity set $\boldsymbol{\xi}_{\alpha}$, equilibrium distributions $f_{\beta}^{(0)}(\rho, \mathbf{u})$, and collision matrix $\Omega_{\alpha \beta}$ are chosen so that moments of slowly varying solutions of (2.1) satisfy the Navier-Stokes equations.

The zeroth and first moments of the discrete Boltzmann equation (2.1) with respect to the particle velocity $\boldsymbol{\xi}_{\alpha}$ give the mass and momentum conservation equations

$$
\partial_{t} \rho+\nabla \cdot(\rho \mathbf{u})=0, \quad \partial_{t}(\rho \mathbf{u})+\nabla \cdot \boldsymbol{\Pi}=0 .
$$

The right hand sides vanish under the assumption that collisions locally conserve mass and momentum, which implies conditions on $f_{\beta}^{(0)}(\rho, \mathbf{u})$ and $\Omega_{\alpha \beta}$. The second moment of (2.1) with respect to $\boldsymbol{\xi}_{\alpha}$ gives an evolution equation for the momentum flux,

$$
\partial_{t} \boldsymbol{\Pi}+\nabla \cdot \mathcal{Q}=-\frac{1}{\tau}\left(\boldsymbol{\Pi}-\boldsymbol{\Pi}^{(0)}\right)
$$

which involves the third moment $\mathcal{Q}$ defined in (2.2). The right hand side of (2.4) arises from $\Pi$ being an eigenfunction of the collision operator with eigenvalue $-1 / \tau$. For example, the Bhatnagar-Gross-Krook [7] or BGK collision matrix $\Omega_{\alpha \beta}=$ $(1 / \tau) \delta_{\alpha \beta}$ has this property. More generally, $\Omega_{\alpha \beta}$ is constructed to have this property by specifying its basis of eigenvectors and their associated eigenvalues $[23,60,19]$. The superscript zero on $\Pi^{(0)}$ in (2.4) indicates a moment of the equilibrium distributions $f_{\beta}^{(0)}(\rho, \mathbf{u})$. These are typically quadratic polynomials in the fluid velocity $\mathbf{u}[55,74]$

$$
f_{\beta}^{(0)}(\rho, \mathbf{u})=\rho w_{\beta}\left(1+\frac{1}{\theta} \mathbf{u} \cdot \boldsymbol{\xi}_{\beta}+\frac{2}{\theta^{2}} \mathbf{u} \mathbf{u}:\left(\boldsymbol{\xi}_{\beta} \boldsymbol{\xi}_{\beta}-\theta \mathbf{I}\right)\right) .
$$

The $w_{\beta}$ are a set of weights associated with the discrete velocities $\boldsymbol{\xi}_{\beta}$. The constant $\theta$ determines the effective temperature in the equilibrium momentum flux $\mathbf{\Pi}^{(0)}=\theta \rho \mathbf{l}+\rho \mathbf{u} \mathbf{u}$, where I is the identity tensor. The speed of sound is thus $c_{\mathrm{S}}=\theta^{1 / 2}$, and the Mach number is $M a=|\mathbf{u}| / c_{\mathrm{s}}$.

The same three equations (2.3) and (2.4) may be derived from the first three integral moments of the continuous Boltzmann equation $[13,15]$. However, in continuous kinetic theory it is more common to use moments with respect to the peculiar velocity, the difference $\mathbf{c}=\boldsymbol{\xi}-\mathbf{u}$ between the particle velocity $\boldsymbol{\xi}$ and the local fluid velocity $\mathbf{u}[38,46,13,15]$. The moments $\boldsymbol{\Pi}$ and $\mathcal{Q}$ may be rewritten in terms of moments with respect to $\mathbf{c}_{\alpha}=\boldsymbol{\xi}_{\alpha}-\mathbf{u}$ as

$$
\Pi_{i j}=P_{i j}+\rho u_{i} u_{j}, \quad \mathcal{Q}_{i j k}=Q_{i j k}+u_{i} P_{j k}+u_{j} P_{k i}+u_{k} P_{i j}+\rho u_{i} u_{j} u_{k} .
$$

The definition of $\mathbf{c}_{\alpha}$ as the discrete peculiar velocity implies $\sum_{\alpha} \mathbf{c}_{\alpha} f_{\alpha}=0$, so all terms with precisely one $\mathbf{c}_{\alpha}$ vanish. The two new quantities appearing in (2.6) are

$$
\mathrm{P}=\sum_{\alpha=0}^{N} \mathbf{c}_{\alpha} \mathbf{c}_{\alpha} f_{\alpha}, \quad \mathrm{Q}=\sum_{\alpha=0}^{N} \mathbf{c}_{\alpha} \mathbf{c}_{\alpha} \mathbf{c}_{\alpha} f_{\alpha}
$$

The left hand side of the momentum flux evolution equation (2.4) becomes

$$
\partial_{t} \Pi_{i j}+\partial_{k} \mathcal{Q}_{i j k}=\partial_{t}\left(P_{i j}+\rho u_{i} u_{j}\right)+\partial_{k}\left(Q_{i j k}+u_{i} P_{j k}+u_{j} P_{i k}+u_{k} P_{i j}+\rho u_{i} u_{j} u_{k}\right),
$$

and we use the mass and momentum conservation equations to evaluate

$$
\begin{aligned}
\partial_{t}\left(\rho u_{i} u_{j}\right) & =u_{i} \partial_{t}\left(\rho u_{j}\right)+u_{j} \partial_{t}\left(\rho u_{i}\right)-u_{i} u_{j} \partial_{t} \rho \\
& =-u_{i} \partial_{k}\left(\rho u_{j} u_{k}+P_{j k}\right)-u_{j} \partial_{k}\left(\rho u_{i} u_{k}+P_{i k}\right)+u_{i} u_{j} \partial_{k}\left(\rho u_{k}\right)
\end{aligned}
$$

Subtracting (2.9) from (2.8) gives an evolution equation for the pressure tensor,

$$
\partial_{t} P_{i j}+\partial_{k}\left(u_{k} P_{i j}+Q_{i j k}\right)+P_{i k} \frac{\partial u_{j}}{\partial x_{k}}+P_{k j} \frac{\partial u_{i}}{\partial x_{k}}=-\frac{1}{\tau}\left(P_{i j}-P_{i j}^{(0)}\right) .
$$



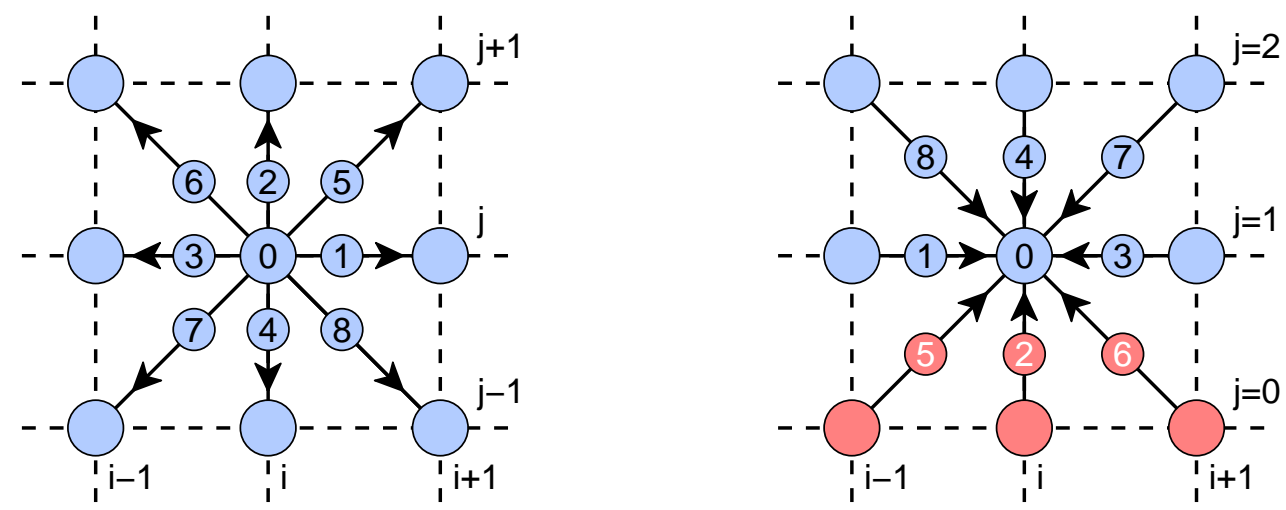

FIG. 2.1. (left) The nine discrete velocities in the D2Q9 lattice. (right) The boundary conditions at the lower edge of the domain must supply values for the three incoming distributions $f_{2}, f_{5}, f_{6}$ as described in Sec. 9.

The same equation may be derived directly from the continuous Boltzmann equation as a special case of Maxwell's equation of transfer for the evolution of an arbitrary moment of $f(\mathbf{x}, \boldsymbol{\xi}, t)$ with respect to $\mathbf{c}=\boldsymbol{\xi}-\mathbf{u}[15]$.

We now isolate the deviatoric stress $\mathrm{T}=\rho \theta \mathrm{I}-\mathrm{P}$, which evolves according to

$$
\partial_{t} T_{i j}+\partial_{k}\left(T_{i j} u_{k}-Q_{i j k}\right)-\rho \theta\left(\frac{\partial u_{i}}{\partial x_{j}}+\frac{\partial u_{j}}{\partial x_{i}}\right)+T_{i k} \frac{\partial u_{j}}{\partial x_{k}}+T_{j k} \frac{\partial u_{i}}{\partial x_{k}}=-\frac{1}{\tau} T_{i j},
$$

assuming the usual isothermal (constant $\theta$ ) equation of state for lattice Boltzmann hydrodynamics. The standard quadratic polynomial equilibria (2.5) give

$$
\mathrm{Q}^{(0)}=-\rho \mathbf{u} \mathbf{u} \mathbf{u}=O\left(M a^{3}\right)
$$

while $\mathrm{Q}^{(0)}=0$ for the continuous Maxwell-Boltzmann distribution. We may design the collision operator $\Omega_{\alpha \beta}$ to apply a very short relaxation time $\tau_{\mathrm{Q}} \ll \tau$ to $\mathrm{Q}$, keeping it near equilibrium, and thus negligibly small. Similarly, we use the low Mach number near-incompressibility condition $\partial_{k} u_{k}=O\left(M a^{2}\right)$ to simplify the $\partial_{k}\left(u_{k} T_{i j}\right)$ term. Making these two approximations in (2.11) gives

$$
T_{i j}+\tau\left[\partial_{t} T_{i j}+u_{k} \partial_{k} T_{i j}+T_{i k} \frac{\partial u_{j}}{\partial x_{k}}+T_{j k} \frac{\partial u_{i}}{\partial x_{k}}\right]=\tau \rho \theta\left(\frac{\partial u_{i}}{\partial x_{j}}+\frac{\partial u_{j}}{\partial x_{i}}\right) .
$$

This closely resembles the upper convected Maxwell model with parameters $\lambda=\tau$ and $\mu=\tau \rho \theta$. Neglecting all the terms multiplied by $\tau$ on the left hand side gives the Navier-Stokes relation $\mathrm{T}=\mu \mathrm{E}$. However, the third and fourth terms in the convected time derivative have the opposite signs from (1.3). This difference, which encapsulates the incompatibility between kinetic theory and the principle of material frame indifference that mandates an objective stress-strain relation, has long been a source of contention between the two fields [94, 26, 10, 52, 32], and was first identified in a lattice Boltzmann context by Wagner [92]. The difference ultimately arises from $\mathcal{Q}$ being a completely symmetric third rank tensor. The three terms $u_{i} P_{j k}$, $u_{j} P_{i k}$, and $u_{k} P_{i j}$ in (2.8) thus all have the same sign, so the $T_{i k} \partial_{k} x_{j}$ and $T_{k j} \partial_{k} u_{i}$ terms in (2.13) have the same sign as the $u_{k} \partial_{k} T_{i j}$ term. By contrast, the upper convected derivative ultimately arises from the $\mathbf{u} \cdot \nabla \boldsymbol{\ell}-\boldsymbol{\ell} \cdot \nabla \mathbf{u}$ combination that describes the stretching of material line elements. This cannot be the evolution equation for the first moment of a scalar distribution function, since the required tensor $\mathbf{u} \ell-\ell \mathbf{u}$ is antisymmetric rather than symmetric [18].

3. Planar channel flow in the kinetic model. We illustrate the consequences of the $T \cdot \nabla \mathbf{u}$ term and its transpose in (2.13) by considering a uni-directional channel flow with $\mathbf{u}=u(y, t) \hat{\boldsymbol{x}}$ in the standard rheological orientation $[85,76,17,35,91,71]$. The stress advection $\mathbf{u} \cdot \nabla \mathrm{T}$ vanishes in this geometry, and the stress evolution equation (2.13) becomes

$$
\mathrm{\top}+\tau\left[\partial_{t} \mathrm{\top}+u^{\prime}\left(\begin{array}{cc}
2 T_{x y} & T_{y y} \\
T_{y y} & 0
\end{array}\right)\right]=\mu\left(\begin{array}{cc}
0 & u^{\prime} \\
u^{\prime} & 0
\end{array}\right)
$$

where $u^{\prime}=\partial_{y} u$, and $\mu=\tau \rho \theta$ as before. The three independent components of (3.1) are

$$
T_{x x}+\tau\left[\partial_{t} T_{x x}+2 u^{\prime} T_{x y}\right]=0, \quad T_{x y}+\tau\left[\partial_{t} T_{x y}+u^{\prime} T_{y y}\right]=\mu u^{\prime}, \quad T_{y y}=0,
$$

and their steady solution is

$$
T_{x x}=-2 \mu \tau u^{\prime 2}, \quad T_{x y}=\mu u^{\prime}, \quad T_{y y}=0 .
$$

The shear stress $T_{x y}$ takes the form one expects from the Navier-Stokes equations, but the non-zero $T_{x x}$ is an $O\left(K n^{2}\right)$ correction to the Navier-Stokes solution. An equivalent term has been found in solutions of the Burnett equations for Poiseuille flow, from an integral representation of the solution of the continuous Boltzmann-BGK equation for Couette flow [96], by 

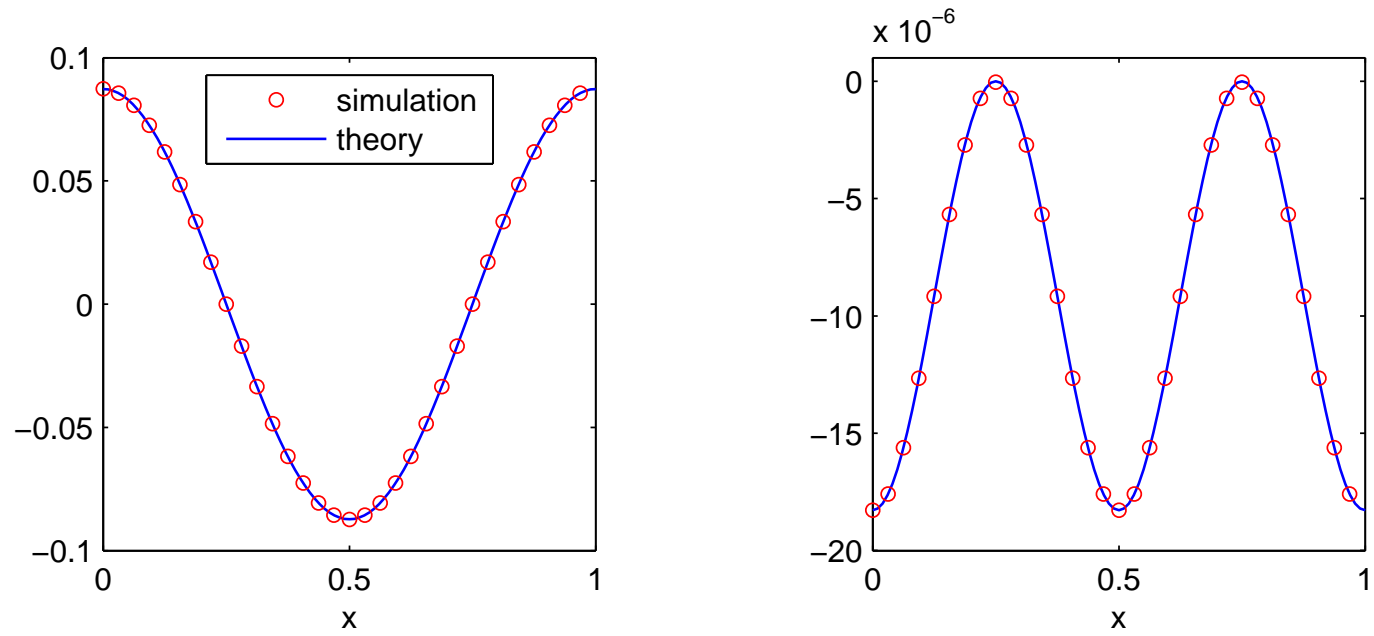

FIG. 3.1. Shear stress $T_{x y}$ (left) and tangential stress $T_{x x}$ (right) in a lattice Boltzmann simulation of freely decaying sinusoidal shear flow compared with the quasistationary theory (3.3). The same legend applies to both plots.

perturbative solutions of the continuous Boltzmann-BGK equation for small forcing [89, 77], and in Direct Simulation Monte Carlo (DSMC) simulations [91, 35].

Figure 3.1 shows the nonzero stress components in a lattice Boltzmann simulation of a freely decaying sinusoidal shear flow starting from initial conditions with $\mathbf{u}=\sin (2 \pi y) \hat{\boldsymbol{x}}$ and $\rho=1$ with viscosity $\nu=0.1$ in the periodic domain $0 \leq y \leq 1$. This simulation avoids the additional complexity of a body force or non-periodic boundary conditions, while the steady solutions (3.3) provide accurate approximations when the viscous decay time for the flow is much longer than the stress relaxation time $\tau$. The simulation employed the D2Q9 lattice shown in figure 2.1, with the weights $w_{0}=4 / 9, w_{1,2,3,4}=1 / 9$ and $w_{5,6,7,8}=1 / 36$. The simulation was run on a lattice of 128 points in $y$ with Mach number $M a=\sqrt{3} / 50$ and the BGK collision matrix $\Omega_{\alpha \beta}=(1 / \tau) \delta_{\alpha \beta}$.

As described in Sec. 5, the lattice Boltzmann equation

$$
\bar{f}_{\alpha}\left(\mathbf{x}+\boldsymbol{\xi}_{\alpha} \Delta t, t+\Delta t\right)=\bar{f}_{\alpha}(\mathbf{x}, t)-\frac{\Delta t}{\tau+\Delta t / 2}\left(\bar{f}_{\alpha}(\mathbf{x}, t)-f_{\alpha}^{(0)}(\mathbf{x}, t)\right)
$$

arises from a space/time discretisation of the discrete Boltzmann equation (2.1) with the BGK collision operator. The BGK collision time $\tau$ is replaced by $\tau+\Delta t / 2$ in the denominator of the right hand side of (3.4), a correction originally derived by Hénon [43] for linear shear flows in lattice gas automata. This correction may also be understood as arising from a CrankNicolson discretisation of the ordinary differential equations $d_{t} f_{\alpha}=-(1 / \tau)\left(f_{\alpha}-f_{\alpha}^{(0)}\right)$ governing collisions in a spatially homogeneous state, while an uncorrected ratio $\Delta t / \tau$ in the right hand side of (3.4) would arise from a forward Euler discretisation [21]. Throughout this work $\tau$ denotes the stress relaxation time in the discrete Boltzmann PDE, so the Newtonian viscosity is always $\mu=\tau \rho \theta$.

The correction of $\tau$ to $\tau+\Delta t / 2$ is accompanied by a transformation of the distribution functions from $f_{\alpha}$ to [40]

$$
\bar{f}_{\alpha}=f_{\alpha}+\frac{\Delta t}{2 \tau}\left(f_{\alpha}-f_{\alpha}^{(0)}\right)
$$

The second moment of this transformation gives an expression for the deviatoric stress in a lattice Boltzmann simulation:

$$
\mathrm{T}=\frac{\boldsymbol{\Pi}^{(0)}-\overline{\boldsymbol{\Pi}}}{1+\Delta t /(2 \tau)}, \quad \text { where } \quad \overline{\boldsymbol{\Pi}}=\sum_{\alpha=0}^{N} \boldsymbol{\xi}_{\alpha} \boldsymbol{\xi}_{\alpha} \bar{f}_{\alpha},
$$

with a corresponding Hénon correction to the denominator. Figure 3.1 shows the reconstructed $T_{x x}$ and $T_{x y}$ at $t=0.5$, by which time the maximum velocity has decayed to $\exp \left(-\pi^{2} / 5\right) \approx 0.139$. Both stress components are in excellent agreement with the theoretical expressions from (3.3) for the instantaneous velocity field $u(y)=\exp \left(-\pi^{2} / 5\right) \sin (2 \pi y)$. In particular, the right hand plot in figure 3.1 confirms the existence and sign of the tangential stress $T_{x x}$ predicted by the kinetic equation, and the fixed relation $\mu=\tau \rho \theta$ between $\mu$ and $\tau$.

4. Coupling to a second local stress tensor. The Jeffreys model expresses the total deviatoric stress as a linear combination of a standard Newtonian viscous stress due to a solvent, and a second viscoelastic stress due to embedded polymer molecules. Following the work of Giraud et al. [36, 37] we therefore seek to generalise the Maxwell-like behavior of the deviatoric stress given by the discrete kinetic models from Sec. 2 by introducing a second stress tensor M that is local to lattice points. However, we do not attempt to represent $M$ explicitly using additional distribution functions, but instead introduce a general linear coupling between $M$ and the existing stress tensor T defined in Sec. 2.

Setting $Q=0$ in the evolution equation (2.11) for the deviatoric stress gives

$$
\mathbb{D} \mathrm{T}-\rho \theta \mathrm{E}=-\frac{1}{\tau} \mathrm{T},
$$


where $\mathbb{D T}$ denotes the convected derivative of $\mathrm{T}$ that appears in (2.11). We now generalise (4.1) to

$$
\left(\begin{array}{c}
\mathbb{D} \mathrm{T}-\rho \theta \mathrm{E} \\
\partial_{t} \mathrm{M}
\end{array}\right)=-\frac{1}{\Lambda}\left(\begin{array}{cc}
a & -1 \\
b & 1
\end{array}\right)\left(\begin{array}{c}
\mathrm{T} \\
\mathrm{M}
\end{array}\right)
$$

where the scalar cofficients in the $2 \times 2$ matrix multiply the tensors $T$ and $M$. The equation for evolving $M$ contains the partial derivative $\partial_{t} \mathrm{M}$, rather than a convected derivative, because we take $\mathrm{M}$ to be local to lattice points in the numerical implementation below. The pre-factor $1 / \Lambda$ sets the relaxation rate for $M$, with the remaining matrix being dimensionless, and fixes the bottom right matrix coefficient to be 1 . The magnitude of $\mathrm{M}$ relative to $\mathrm{T}$ is arbitrary in a linear theory, so we may also set the upper right matrix coefficient to be -1 .

We now eliminate $\mathrm{M}$ by applying $\left(1+\Lambda \partial_{t}\right)$ to the equation for $\mathrm{T}$ given by the upper row of (4.2),

$$
\mathbb{D T}-\rho \theta \mathrm{E}+\Lambda \partial_{t}(\mathbb{D T}-\rho \theta \mathrm{E})=-\frac{a+b}{\Lambda} \mathrm{T}-a \partial_{t} \mathrm{~T} .
$$

Assigning

$$
a=\lambda / \tau-1, \quad b=1+(\Lambda-\lambda) / \tau, \quad \mu=\tau \rho \theta,
$$

gives

$$
\mathrm{T}+\left[(\lambda-\tau) \partial_{t} \mathrm{~T}+\tau \mathbb{D} \mathrm{T}\right]+\Lambda \tau \partial_{t} \mathbb{D} \mathrm{T}=\mu\left(\mathrm{E}+\Lambda \partial_{t} \mathrm{E}\right)
$$

This is the Burgers viscoelastic model [71], though with a mix of partial and convected time derivatives. The timescale $\tau$ controlling the steady-state viscosity scales with Mach number, due to the relation $\mu=\tau \rho \theta$, while $\lambda$ and $\Lambda$ have no such scaling. At sufficiently small Mach number (ensuring $\tau \ll \Lambda<\lambda$ ) we thus recover the linear Jeffreys model

$$
\mathrm{T}+\lambda \partial_{t} \mathrm{~T}=\mu\left(\mathrm{E}+\Lambda \partial_{t} \mathrm{E}\right)
$$

with a simple partial derivative $\partial_{t} \mathrm{~T}$ instead of the earlier nonlinear convected time derivative $\mathbb{D} T$. The coefficients $a$ and $b$ in the matrix (4.2) are are uniquely determined by the three timescales $\tau, \lambda, \Lambda$ in the Jeffreys model. The eigenvalues of this matrix are

$$
\sigma_{ \pm}=\frac{-\lambda \pm \sqrt{\lambda^{2}-4 \Lambda \tau}}{2 \Lambda \tau}
$$

which are purely real when $4 \Lambda \tau<\lambda^{2}$. The real parts of $\sigma_{ \pm}$are always negative, so (4.2) is a viable model of stress relaxation.

5. From discrete Boltzmann to lattice Boltzmann. We now construct a second-order accurate discretization of the above kinetic equation using operator splitting [21]. The discrete Boltzmann equation (2.1) may be split into separate equations for streaming and collisions,

$$
\partial_{t} f_{\alpha}+\boldsymbol{\xi}_{\alpha} \cdot \nabla f_{\alpha}=0, \quad \partial_{t} f_{\alpha}=-\sum_{\beta=0}^{N} \Omega_{\alpha \beta}\left(f_{\beta}-f_{\beta}^{(0)}\right) .
$$

The first of the pair describes advection along characteristics. Its solution over a timestep $\Delta t$ may be written symbolically as $f_{\alpha}(\mathbf{x}, t+\Delta t)=\mathrm{S} f_{\alpha}(\mathbf{x}, t)=f_{\alpha}\left(\mathbf{x}-\boldsymbol{\xi}_{\alpha} \Delta t, t\right)$ in terms of the streaming operator S. Approximating the solution of the second equation over a timestep $\Delta t$ by the Crank-Nicolson formula gives

$$
\frac{\mathbf{f}(\mathbf{x}, t+\Delta t)-\mathbf{f}(\mathbf{x}, t)}{\Delta t}=-\frac{1}{2} \boldsymbol{\Omega}\left(\mathbf{f}(t+\Delta t)-\mathbf{f}^{(0)}(t+\Delta t)+\mathbf{f}(t)-\mathbf{f}^{(0)}(t)\right),
$$

in matrix notation where $\mathbf{f}=\left(f_{0}, f_{1}, \ldots f_{N}\right)^{\top}$ is a column vector of distribution functions, and $\boldsymbol{\Omega}$ is the collision matrix with components $\Omega_{\alpha \beta}$. The equilibrium distributions $\mathbf{f}^{(0)}(\rho, \mathbf{u})$ are invariant under collisions, since $\rho$ and $\mathbf{u}$ are invariant under collisions, so we may replace $\mathbf{f}^{(0)}(t+\Delta t)$ by $\mathbf{f}^{(0)}(t)$ in $(5.2)$. The solution may then be written as

$$
\mathbf{f}(\mathbf{x}, t+\Delta t)=\mathbf{f}(\mathbf{x}, t)-\widetilde{\Omega}\left(\mathbf{f}(\mathbf{x}, t)-\mathbf{f}^{(0)}(\mathbf{x}, t)\right)
$$

where $\widetilde{\Omega}=\left(\mathrm{I}+\frac{1}{2} \Delta t \Omega\right)^{-1} \Delta t \Omega$ is a discrete collision matrix constructed from the continuous collision matrix $\Omega$. We write the solution (5.3) symbolically as $\mathbf{f}(\mathbf{x}, t+\Delta t)=\mathbf{C} \mathbf{f}(\mathbf{x}, t)$.

We now combine the solution operators $S$ and $C$ using the Strang splitting formula [84]

$$
\mathbf{f}(\mathbf{x}, t+\Delta t)=\mathrm{C}^{1 / 2} \mathrm{SC}^{1 / 2} \mathbf{f}(\mathbf{x}, t)
$$

where $C^{1 / 2}$ denotes the action of the collision operator for a half-timestep of length $\Delta t / 2$. This symmetric splitting gives a second order in $\Delta t$ approximation to the evolution under the unsplit discrete Boltzmann equation (2.1). For linear operators $\mathrm{S}$ and $\mathrm{C}$ this splitting formula is a consequence of the Baker-Cambell-Hausdorff and Zassenhaus formulae for the exponential 
of a sum of non-commuting operators. Its nonlinear extension may be accomplished using the notion of the Lie derivative of a nonlinear operator [39].

Applying the Strang splitting formula repeatedly for $n$ timesteps gives

$$
\mathbf{f}(\mathbf{x}, t+n \Delta t)=\mathrm{C}^{1 / 2} \mathrm{SC}^{1 / 2} \mathrm{C}^{1 / 2} \mathrm{SC}^{1 / 2} \ldots \mathrm{C}^{1 / 2} \mathrm{SC}^{1 / 2} \mathbf{f}(\mathbf{x}, t),
$$

which simplifies to

$$
\mathbf{f}(\mathbf{x}, t+n \Delta t)=\mathrm{C}^{1 / 2}(\mathrm{~S} \mathrm{C})^{n} \mathrm{C}^{-1 / 2} \mathbf{f}(\mathbf{x}, t),
$$

after using $\mathrm{C}^{1 / 2} \mathrm{C}^{1 / 2}=\mathrm{C}$ and $\mathrm{C}^{1 / 2}=\mathrm{C}^{-1 / 2}$ to combine intermediate stages.

Equation (5.6) for $n=1$ may be rewritten as the standard lattice Boltzmann equation

$$
\bar{f}_{\alpha}\left(\mathbf{x}+\boldsymbol{\xi}_{\alpha} \Delta t, t+\Delta t\right)=\bar{f}_{\alpha}(\mathbf{x}, t)-\sum_{\beta=0}^{N} \widetilde{\Omega}_{\alpha \beta}\left(\bar{f}_{\beta}(\mathbf{x}, t)-f_{\beta}^{(0)}(\mathbf{x}, t)\right)
$$

for the discrete collision matrix $\widetilde{\Omega}$, and the transformed distribution functions $\overline{\mathbf{f}}$ defined by

$$
\overline{\mathbf{f}}=\mathrm{C}^{-1 / 2} \mathbf{f}, \quad \mathbf{f}=\mathrm{C}^{1 / 2} \overline{\mathbf{f}} .
$$

If we take $C^{1 / 2}=\frac{1}{2}(I+C)$, and $C^{-1 / 2}=2(I+C)^{-1}$ to be its exact inverse, we recover the transformation introduced by He $e t$ al. [40] for the single-relaxation-time collision operator $\Omega_{\alpha \beta}=(1 / \tau) \delta_{\alpha \beta}$, and extended to general matrix collision operators by Dellar [19]. The replacement of $\Omega$ by $\widetilde{\Omega}=\left(\mathrm{I}+\frac{1}{2} \Delta t \Omega\right)^{-1} \Delta t \Omega$ in the Crank-Nicolson definition of $\mathrm{C}$ generalises the Hénon correction in Sec. 3 that replaces the single relaxation time $\tau$ in the discrete Boltzmann-BGK PDE with $\tau+\Delta t / 2$ in the lattice Boltzmann equation [43].

6. Viscoelastic implementation. The above formulation extends easily to encompass coupling to a second set of variables, the components of $\mathrm{M}$ located at each lattice point, that only take part in the collision step. These variables are thus invariant under the streaming step. To construct the viscoelastic collision step it is beneficial to replace $\Pi$ with $T=\Pi \Pi^{(0)}-\Pi$. The equilibrium momentum flux $\mathbf{\Pi}^{(0)}=\theta \rho \mathbf{I}+\rho \mathbf{u} \mathbf{u}$ is invariant under collisions, being a function of $\rho$ and $\mathbf{u}$, so the postcollisional momentum flux $\boldsymbol{\Pi}^{\prime}=\boldsymbol{\Pi}^{(0)}-\boldsymbol{T}^{\prime}$ may be easily reconstructed from the post-collisional deviatoric stress $\mathbf{T}^{\prime}$. The simplest approach reconstructs the post-collisional distribution functions from the truncated Hermite expansion [41]

$$
\bar{f}_{\alpha}^{\prime}=w_{\alpha}\left[\rho+3 \rho \mathbf{u} \cdot \boldsymbol{\xi}_{\alpha}+\frac{9}{2}\left(\rho \mathbf{u u}-\overline{\mathbf{T}}^{\prime}\right):\left(\boldsymbol{\xi}_{\alpha} \boldsymbol{\xi}_{\alpha}-\frac{1}{3} \mathbf{I}\right)\right],
$$

for lattices with $\theta=1 / 3$, and propagates them to adjacent lattice points

$$
\bar{f}_{\alpha}\left(\mathbf{x}+\boldsymbol{\xi}_{\alpha} \Delta t, t+\Delta t\right)=\bar{f}_{\alpha}^{\prime}(\mathbf{x}, t) .
$$

The overbars indicate the transformed distribution functions defined by (5.8) and their corresponding moments such as $\overline{\mathrm{T}}$, while the fluid density $\rho$ and velocity $\mathbf{u}$ are unaffected by this transformation. The expansion (6.1) coincides with the standard quadratic equilibria when $\overline{\mathrm{T}}^{\prime}=0$. It implicitly resets the higher, non-hydrodynamic, or "ghost" moments of the distribution function to their equilibrium values at every timestep [44, 58, 66, 19]. This is equivalent to applying a continuous relaxation time $\tau_{\text {ghost }}=\Delta t / 2$ to these moments. Alternatively, the relaxation times for these moments may be freely chosen in the usual way $[23,60]$, independently of the coupling between the stress moment $T$ and the second stress $M$

The construction of a viscoelastic lattice Boltzmann algorithm thus reduces to constructing the post-collisional stress $\overline{\mathrm{T}}^{\prime}$ in the transformed $\bar{f}_{\alpha}$ variables. The general formula (5.3) gives the discrete analog of the matrix in (4.2) as

$$
\mathrm{C}=\frac{1}{4 \Lambda \tau+2 \lambda \Delta t+\Delta t^{2}}\left(\begin{array}{cc}
4 \Lambda \tau+2 \Delta t(2 \tau-\lambda)-\Delta t^{2} & 4 \tau \Delta t \\
4 \Delta t(\lambda-\Lambda-\tau) & 4 \Lambda \tau+2 \Delta t(\lambda-2 \tau)-\Delta t^{2}
\end{array}\right)
$$

The eigenvalues of this matrix,

$$
\tilde{\sigma}_{ \pm}=\frac{4 \Lambda \tau-\Delta t^{2} \pm 2 \Delta t\left(\lambda^{2}-4 \tau \Lambda\right)^{1 / 2}}{4 \Lambda \tau+2 \lambda \Delta t+\Delta t^{2}},
$$

are real when $4 \tau \Lambda<\lambda^{2}$, and otherwise complex. The eigenvalues always lie within the unit circle, since

$$
\left(4 \Lambda \tau-\Delta t^{2}\right)^{2}+4 \Delta t^{2}\left(4 \tau \Lambda-\lambda^{2}\right)<\left(4 \Lambda \tau+2 \lambda \Delta t+\Delta t^{2}\right)^{2}
$$

whenever $\lambda \Delta t>0$. This discrete collision step is thus linearly stable for all positive values of the parameters $\mu, \lambda, \Lambda$ in the Jeffreys fluid model.

The collision matrix $C$ is applied component-by-component to the the transformed stress tensors $\overline{\mathrm{T}}$ and $\overline{\mathrm{M}}$ defined by

$$
\left(\frac{\bar{T}}{\bar{M}}\right)=2(I+C)^{-1}\left(\begin{array}{c}
T \\
M
\end{array}\right) \text {. }
$$

The $2 \times 2$ matrices $\mathrm{C}$ and $(\mathrm{I}+\mathrm{C})^{-1}$ act either on the whole tensors $\mathrm{T}$ and $\mathrm{M}$, or just on their traceless parts. The latter is sufficient for linear viscoelasticity, and is easily accomplished by decomposing $\overline{\mathrm{T}}$ into its trace $\operatorname{Tr} \overline{\mathrm{T}}=\bar{T}_{x x}+\bar{T}_{y y}+\bar{T}_{z z}$, its off-diagonal components $\bar{T}_{x y}, \bar{T}_{x z}, \bar{T}_{y z}$, and the normal stress differences $\bar{T}_{x x}-\bar{T}_{y y}$ and $\bar{T}_{y y}-\bar{T}_{z z}$. We thus need two extra degrees of freedom per lattice point for the traceless part of $\overline{\mathrm{M}}$ in two dimensions, and five extra degrees of freedom per lattice point in three dimensions. 

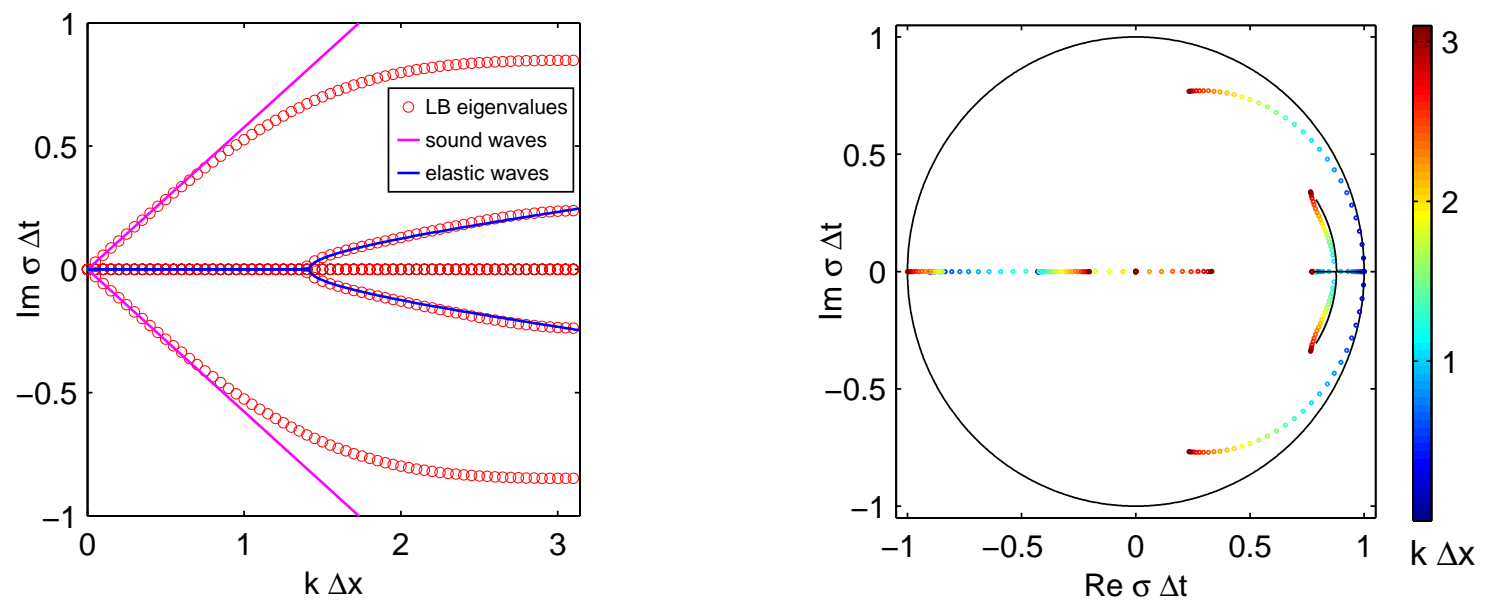

FIG. 7.1. Imaginary parts (left) and complex values (right) of the eigenvalues for axis-aligned sinusoidal disturbances with wavenumber $k$. The continuous curves in the left plot show the theoretical dispersion relations $\operatorname{Im} \sigma= \pm c_{\mathrm{S}} k$ for acoustic waves and (7.2) for elastic waves. The continuous curves in the right plot show the theoretical dispersion relation for elastic waves and the unit circle.

7. Dispersion relations for linear waves. The properties of lattice Boltzmann algorithms are often studied using the von Neumann approach that seeks plane wave solutions to the linearised form of discrete equations such as (3.4) and its viscoelastic generalisations $[81,36,60,59]$. Unsteady shear flow in an incompressible Jeffreys fluid is governed by the coupled linear equations

$$
\rho_{0} \partial_{t} u=\partial_{y} T, \quad T+\lambda \partial_{t} T=\mu\left(\partial_{y} u+\Lambda \partial_{t y} u\right)
$$

where $\mathbf{u}=u(y, t) \hat{\boldsymbol{x}}$ in the standard rheological orientation of Sec. 3, and $T=T_{x y}$. Solutions proportional to $\exp (i k y+\sigma t)$ exist when the growth rate $\sigma$ satisfies the dispersion relation

$$
\sigma=-\frac{1}{2 \lambda}\left[1+\Lambda \nu k^{2} \pm\left(\left(1+\Lambda \nu k^{2}\right)^{2}-4 \lambda \nu k^{2}\right)^{1 / 2}\right] .
$$

This reduces to the expected purely viscous relation $\sigma=-\nu k^{2}$ in the double limit as $\lambda$ and $\Lambda$ tend to zero. The dispersion relation for a Maxwell fluid $(\Lambda=0)$ takes the simpler form

$$
\sigma=-\frac{1}{2 \lambda}\left[1 \pm\left(1-4 \lambda \nu k^{2}\right)^{1 / 2}\right] .
$$

Both dispersion relations imply $\operatorname{Re} \sigma<0$ for all parameter values, so disturbances are damped by viscosity. However, $\sigma$ may become complex, showing that the elastic property of the fluid may support oscillations in the form of decaying transverse shear waves. Disturbances in a Maxwell fluid become oscillatory if $4 \lambda \nu k^{2}>1$, while disturbances in a Jeffreys fluid become oscillatory in the band of wavenumbers $k$ for which $4 \lambda \nu k^{2}>\left(1+\Lambda \nu k^{2}\right)^{2}$.

Seeking solutions to the numerical algorithm described in Secs. 5 and 6 for sinusoidal disturbances with amplitude $\epsilon \ll 1$ about a uniform rest state in the form $\bar{f}_{\alpha}=w_{\alpha}+\epsilon h_{\alpha} \exp (i \mathbf{k} \cdot \mathbf{x}+\sigma t)$ and $\overline{\mathrm{M}}=\epsilon \mathrm{H} \exp (i \mathbf{k} \cdot \mathbf{x}+\sigma t)$ gives a matrix eigenvalue problem for the constants $h_{\alpha}$ and $\mathrm{H}$. Combining the D2Q9 lattice Boltzmann model from Sec. 3 with a traceless stress perturbation $\mathrm{H}$ represented by $H_{x y}$ and $H_{n}=H_{x x}-H_{y y}$ gives 11 degrees of freedom in total. The resulting linear system may be written as

$$
\begin{aligned}
e^{\sigma \Delta t} & e^{i \boldsymbol{\xi}_{\alpha} \cdot \mathbf{k} \Delta x} h_{\alpha}=h_{\alpha}-\sum_{\beta=0}^{8} L_{\alpha \beta}(\tau) h_{\beta} \\
& +9 w_{\alpha}\left(\xi_{\alpha x}^{2}-\xi_{\alpha y}^{2}\right)\left[(\lambda-\tau+\Delta t / 2)\left(-h_{1}+h_{2}-h_{3}+h_{4}\right)-\tau H_{n}\right] / \Delta \\
& +36 w_{\alpha} \xi_{\alpha x} \xi_{\alpha y}\left[(\lambda-\tau+\Delta t / 2)\left(-h_{5}+h_{6}-h_{7}+h_{8}\right)-\tau H_{x y}\right] / \Delta, \\
e^{\sigma \Delta t} H_{n} & =H_{n}-4\left[(\Lambda-\lambda+\tau)\left(-h_{1}+h_{2}-h_{3}+h_{4}\right)+(\tau+\Delta t / 2) H_{n}\right] / \Delta, \\
e^{\sigma \Delta t} H_{x y} & =H_{x y}-4\left[(\Lambda-\lambda+\tau)\left(-h_{5}+h_{6}-h_{7}+h_{8}\right)+(\tau+\Delta t / 2) H_{x y}\right] / \Delta,
\end{aligned}
$$

where $\Delta=\Delta t+2 \tau+4 \tau \Lambda / \Delta t$, and $L_{\alpha \beta}(\tau)$ is the usual linearised hydrodynamic collision operator. This system defines an $11 \times 11$ matrix eigenvalue problem for the eigenvalues $e^{\sigma \Delta t}$ and their corresponding eigenvectors $\left(h_{0}, \ldots h_{8}, H_{n}, H_{x y}\right)$.

Figure 7.1 shows the 11 eigenvalues $e^{\sigma \Delta t}$ as a function of $k \Delta x$ for disturbances with wave vector $\mathbf{k}=(k, 0)$ aligned with the $x$-axis. The normalisation of lengths with $\Delta x$ and times with $\Delta t$ is convenient for investigating properties of the algorithm on the lattice scale, and is equivalent to working in the so-called lattice units in which $\Delta x=1$ and $\Delta t=1$. The parameter values $\tau=0.1 \Delta t, \lambda=4 \Delta t$ and $\Lambda=0.5 \Delta t$ are chosen to show the transverse elastic waves on the same axes as the longitudinal acoustic waves that propagate at the sound speed $c_{\mathrm{s}}=\sqrt{1 / 3}(\Delta x / \Delta t)$. The eigenvalues of the discrete algorithm corresponding to the elastic waves are in good agreement with the theoretical dispersion relation (7.2) for a Jeffreys 

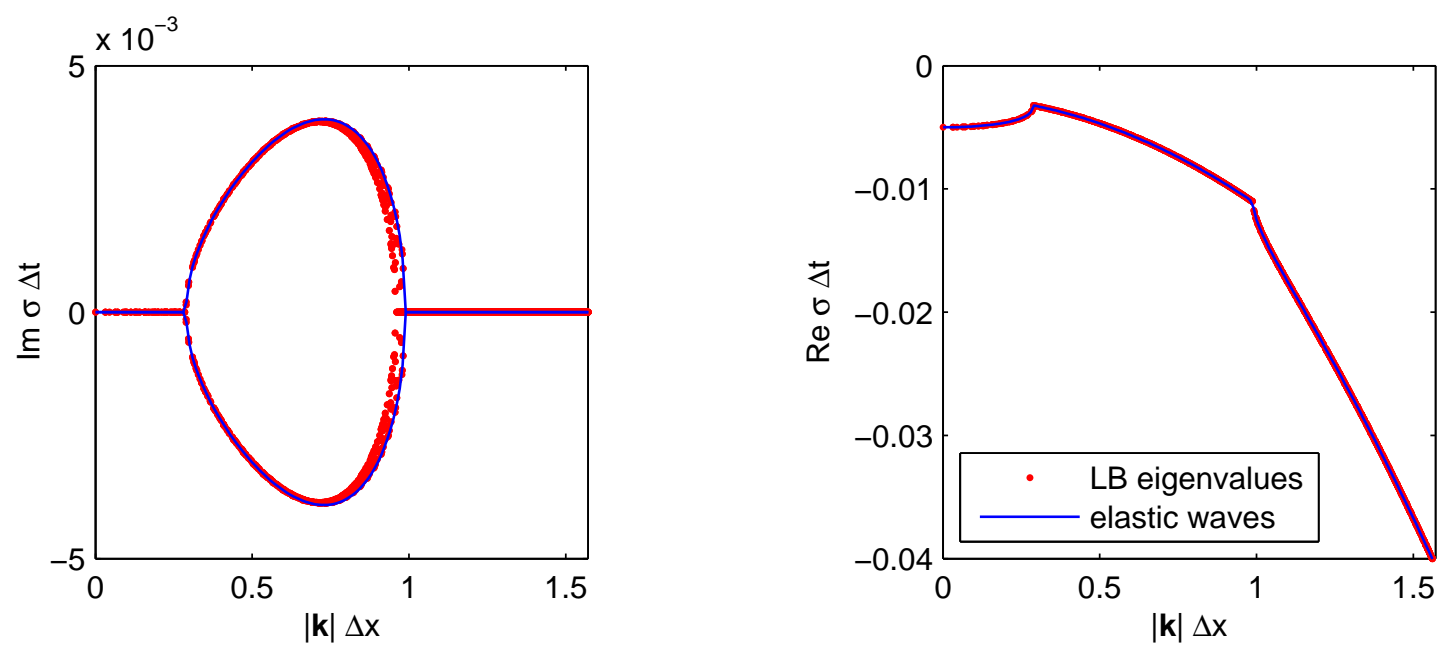

FIG. 7.2. Scatter plots of the wave speeds (left) and decay rates (right) for numerical transverse elastic waves with wavevector $\mathbf{k}$ and parameters $\lambda=200 \Delta t, \Lambda=140 \Delta t, \tau=0.075 \Delta t$. The same key applies to both plots, with the solid lines showing the elastic wave dispersion relation (7.2). The sound waves are not visible on these axes.

fluid, although the the large wavenumber cut-off for the elastic waves is not visible because it lies beyond the largest resolved wavenumber with $k \Delta x=\pi$.

However, the convergence of the discrete algorithm towards solutions of the PDEs describing an incompressible Jeffreys fluid requires $k \Delta x \rightarrow 0$, and an asymptotic separation between the acoustic and elastic wave speeds. Figure 7.2 shows the real and imaginary parts of the eigenvalues for the more realistic parameter values $\tau=0.075 \Delta t, \lambda=200 \Delta t, \Lambda=140 \Delta t$, computed for a grid of wave vectors $\mathbf{k}=\left(k_{x}, k_{y}\right)$ for which $k_{x} \Delta x$ and $k_{y} \Delta x$ both lie in the set $\{\pi / 100,2 \pi / 100, \ldots, 99 \pi / 100\}$. The near-perfect collapse of these data points onto single curves when plotted against $|\mathbf{k}|$ demonstrates the isotropy of the algorithm. The finite band of wavenumbers for which elastic waves exist in the Jeffreys model is now visible, but the much faster acoustic waves are not visible on these axes. The phase speeds in the left-hand plot are noticably affected by the finite spatial resolution for $|\mathbf{k}| \Delta x \approx 1$. Small errors in the effective numerical wavenumber become visible here because the derivative $d \sigma / d k$ of the theoretical solution (7.2) becomes infinite at the points where $\operatorname{Im} \sigma$ crosses zero.

8. Implementation of a body force. A wider range of benchmark flows may be simulated by including a body force $\mathbf{F}$ in the momentum equation. The continuous Boltzmann equation for a distribution $f(\mathbf{x}, \boldsymbol{\xi}, t)$ of particles each experiencing an acceleration a due to external body forces is

$$
\partial_{t} f+\boldsymbol{\xi} \cdot \nabla f+\mathbf{a} \cdot \nabla_{\boldsymbol{\xi}} f=C[f, f]
$$

where the right hand side is Boltzmann's binary collision operator $[13,15]$. The first three moments of $(8.1)$ give

$$
\begin{aligned}
\partial_{t} \rho+\nabla \cdot(\rho \mathbf{u}) & =0 \\
\partial_{t}(\rho \mathbf{u})+\nabla \cdot \boldsymbol{\Pi} & =\mathbf{F}, \\
\partial_{t} \boldsymbol{\Pi}+\nabla \cdot \mathbf{Q} & =-\frac{1}{\tau}\left(\boldsymbol{\Pi}-\boldsymbol{\Pi}^{(0)}\right)+\mathbf{F} \mathbf{u}+\mathbf{u} \mathbf{F},
\end{aligned}
$$

where $\mathbf{F}=\rho \mathbf{a}$. The moments of the acceleration term $\mathbf{a} \cdot \nabla_{\boldsymbol{\xi}} f$ have been written on the right hand sides, as is conventional for fluid equations. The body force does not appear in the continuity equation, and appears as expected in the momentum equation. The additional terms $\mathbf{F} \mathbf{u}+\mathbf{u} \mathbf{F}$ on the right hand side of (8.2c) ensure that the body force disappears from the evolution equation for the stress $T$. The body force modifies the previous equation (2.9) to

$$
\partial_{t}\left(\rho u_{i} u_{j}\right)=u_{i} F_{j}-u_{i} \partial_{k}\left(\rho u_{j} u_{k}+P_{j k}\right)+u_{j} F_{i}-u_{j} \partial_{k}\left(\rho u_{i} u_{k}+P_{i k}\right)+u_{i} u_{j} \partial_{k}\left(\rho u_{k}\right),
$$

with additional terms $u_{i} F_{j}$ and $u_{j} F_{i}$. These cancel with the matching terms on the right hand side of (8.2c) as rewritten using $\mathrm{P}$,

$$
\partial_{t}\left(P_{i j}+\rho u_{i} u_{j}\right)+\partial_{k}\left(Q_{i j k}+u_{i} P_{j k}+u_{j} P_{i k}+u_{k} P_{i j}+\rho u_{i} u_{j} u_{k}\right)
$$

$$
=-\frac{1}{\tau}\left(P_{i j}-P_{i j}^{(0)}\right)+u_{i} F_{j}+u_{j} F_{i}
$$

to leave the unmodified equations (2.10) and (2.13) for $\mathrm{P}$ and $\mathrm{T}$.

The combination of the splitting approach of Sec. 5 with a representation in terms of moments leads us to discretise the 
ordinary differential equations

$$
\begin{aligned}
\partial_{t} \rho & =0 \\
\partial_{t}(\rho \mathbf{u}) & =\mathbf{F} \\
\partial_{t} \boldsymbol{\Pi} & =-\frac{1}{\tau}\left(\boldsymbol{\Pi}-\mathbf{\Pi}^{(0)}\right)+\mathbf{F} \mathbf{u}+\mathbf{u} \mathbf{F},
\end{aligned}
$$

using the Crank-Nicolson formula. Taking $\mathbf{F}$ to be independent of time, we obtain $\rho^{\prime}=\rho$ and $\mathbf{u}^{\prime}=\mathbf{u}+\rho^{-1} \Delta t \mathbf{F}$. A prime denotes a quantity evaluated at $t+\Delta t$, while unprimed quantities are evaluated at $t$. Applying the Crank-Nicolson formula to $(8.5 \mathrm{c})$ gives

$$
\boldsymbol{\Pi}^{\prime}-\boldsymbol{\Pi}=-\frac{\Delta t}{2 \tau}\left(\boldsymbol{\Pi}^{\prime}+\boldsymbol{\Pi}-\boldsymbol{\Pi}^{\prime(0)}-\boldsymbol{\Pi}^{(0)}\right)+\frac{1}{2} \Delta t\left(\mathbf{F} \mathbf{u}^{\prime}+\mathbf{u}^{\prime} \mathbf{F}+\mathbf{F} \mathbf{u}+\mathbf{u} \mathbf{F}\right)
$$

The last term simplifies using $\mathbf{u}^{\prime}=\mathbf{u}+\rho^{-1} \Delta t \mathbf{F}$,

$$
\frac{1}{2} \Delta t\left(\mathbf{F} \mathbf{u}^{\prime}+\mathbf{u}^{\prime} \mathbf{F}+\mathbf{F} \mathbf{u}+\mathbf{u} \mathbf{F}\right)=\Delta t\left(\mathbf{F} \mathbf{u}+\mathbf{u} \mathbf{F}+\rho^{-1} \Delta t \mathbf{F} \mathbf{F}\right)=\mathbf{\Pi}^{\prime(0)}-\mathbf{\Pi}^{(0)},
$$

so (8.6) becomes

$$
\boldsymbol{\Pi}^{\prime}=\boldsymbol{\Pi}^{(0)}+\left(\frac{\tau-\Delta t / 2}{\tau+\Delta t / 2}\right)\left(\boldsymbol{\Pi}-\boldsymbol{\Pi}^{(0)}\right) .
$$

Converting these expressions for $\rho^{\prime}, \mathbf{u}^{\prime}$ and $\mathbf{\Pi}^{\prime}$ into an expression for the post-collisional distribution functions gives the socalled "exact difference method" [56, 57]

$$
f_{\alpha}^{\prime}=f_{\alpha}-\frac{\Delta t}{\tau+\Delta t / 2}\left(f_{\alpha}-f_{\alpha}^{(0)}\right)+f_{\alpha}^{\prime(0)}-f_{\alpha}^{(0)}
$$

since the contribution from the body force appears solely through the difference $f_{\alpha}^{\prime(0)}-f_{\alpha}^{(0)}$.

Equation (8.8) is exactly the same formula that relates the post-collisional deviatoric stress $\mathbf{T}^{\prime}=\Pi^{\prime(0)}-\Pi^{\prime}$ to the precollisional deviatoric stress $T=\Pi^{(0)}-\Pi$ in the absence of a body force. The body force only contributes through the difference between $\Pi^{\prime(0)}$ and $\Pi^{(0)}$. This decoupling enables the simple inclusion of a body force in the viscoelastic collision algorithm of Sec. 6. We first calculate $T=\Pi^{(0)}-\Pi$, apply the existing algorithm to calculate the corresponding $T^{\prime}$, then finally calculate the post-collisional momentum flux as $\Pi^{\prime}=\Pi^{\prime(0)}-\mathbf{T}^{\prime}$. Second-order accuracy is achieved, as in Sec. 5, by defining barred variables using a half timestep of this collision algorithm. The collision operator defined by (8.9) no longer conserves momentum, since $\mathbf{u}^{\prime}=\mathbf{u}+\rho^{-1} \Delta t \mathbf{F}$, so the $f_{\alpha}$ to $\bar{f}_{\alpha}$ transformation in (5.8) implies [40]

$$
\rho \overline{\mathbf{u}}=\sum_{\alpha=0}^{N} \boldsymbol{\xi}_{\alpha} \bar{f}_{\alpha}=\rho \mathbf{u}-\frac{1}{2} \Delta t \mathbf{F} .
$$

9. Flow due to a tangentially oscillating wall. The flow driven by a tangentially oscillating wall in a Newtonian viscous fluid is known as Stokes' second problem [83]. After an initial transient, the fluid oscillates with the same frequency as the wall, and with an amplitude that decays exponentially with distance from the wall $[4,61]$. This is the spatial analog of the temporally decaying transverse waves studied in Sec. 7. The non-transient part of the solution is given by

$$
u(y, t)=U_{0} \sin (\omega t-k y) e^{-\kappa y},
$$

for a wall moving tangentially with velocity $U_{\text {wall }}=U_{0} \sin (\omega t)$ in the $\hat{x}$ direction. Choosing $\sin (\omega t)$ gives a continuous transition from a rest state for $t<0$ to a moving state for $t>0$, eliminating the generalised functions that appear in the initial transient due to an impulsive start [17]. The wavenumber $k$ and attenuation scale $\kappa$ for the Jeffreys model are [28, 27]

$$
k=\left[\frac{\omega}{2 \mu} \frac{\sqrt{1+\lambda^{2} \omega^{2}} \sqrt{1+\Lambda^{2} \omega^{2}}+(\lambda-\Lambda) \omega}{1+\Lambda^{2} \omega^{2}}\right]^{1 / 2}, \quad \kappa=\frac{\omega}{2 \mu k} \frac{1+\Lambda \lambda \omega^{2}}{1+\Lambda^{2} \omega^{2}}
$$

and the shear stress is

$$
T_{x y}=-U_{0} \frac{\omega}{\sqrt{k^{2}+\kappa^{2}}} \cos \left[\omega t-k y+\tan ^{-1}(k / \kappa)\right] e^{-\kappa y} .
$$

To simulate this flow, the lattice Boltzmann formulation derived above must be supplemented with boundary conditions for the incoming distribution functions $f_{4}, f_{7}, f_{8}$ on the upper boundary at $y=1$, and for $f_{2}, f_{5}, f_{6}$ on the lower boundary at $y=0$ shown in figure 2.1. We use the approach of Wagner \& Yeomans [93] and Bennett [5, 6, 75] to impose boundary conditions on the hydrodynamic moments $u_{x}, u_{y}$ and $\Pi_{x x}$,

$$
\begin{aligned}
\rho U_{\text {wall }} & =\rho u_{x}=f_{1}-f_{3}+f_{5}-f_{6}-f_{7}+f_{8}, \\
0 & =\rho u_{y}=f_{2}-f_{4}+f_{5}+f_{6}-f_{7}-f_{8}, \\
\Pi_{x x}^{(0)} & =\Pi_{x x}=f_{1}+f_{3}+f_{5}+f_{6}+f_{7}+f_{8} .
\end{aligned}
$$



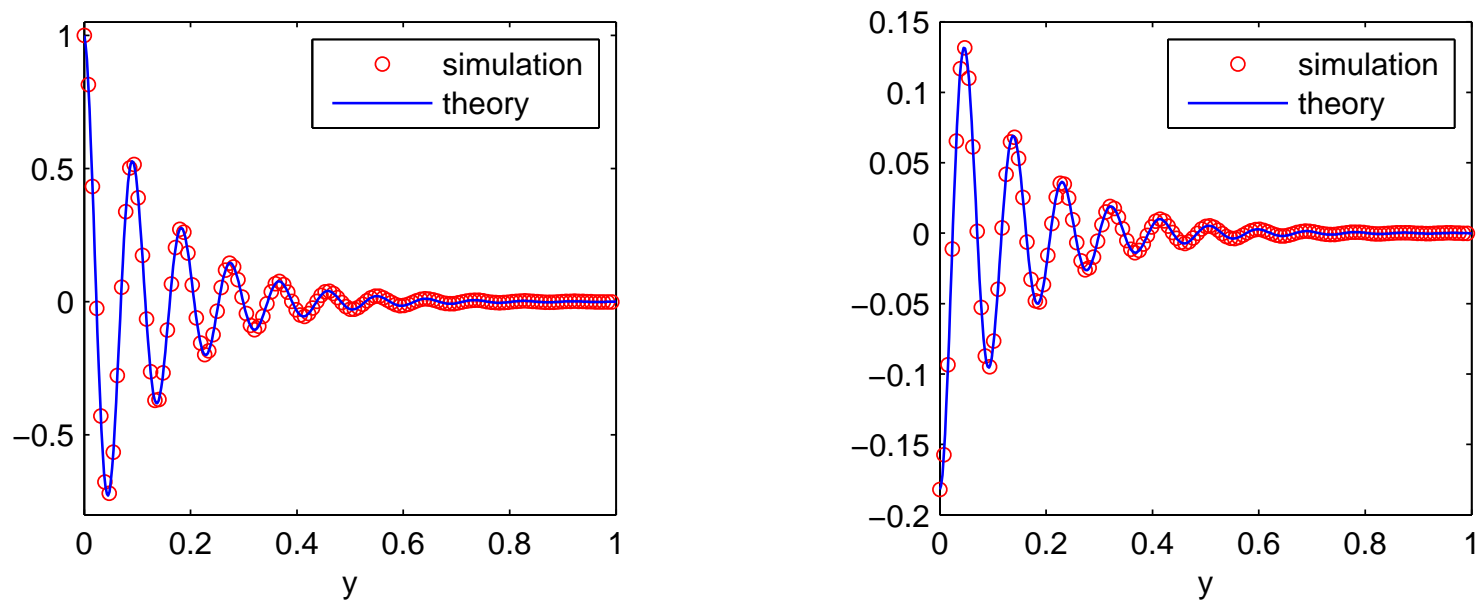

FIG. 9.1. Velocity (left) and shear stress (right) in the spatially decaying sinusoidal shear flow driven by an oscillating wall at $y=0$. The fields are shown at a time of maximum displacement of the wall.

These three moments are chosen because they contain the three linearly independent combinations $f_{5}-f_{6}, f_{2}+f_{5}+f_{6}$, and $f_{5}+f_{6}$, of the unknowns $f_{2}, f_{5}, f_{6}$. The first two conditions (9.4a) and (9.4b) impose no-flux and no-slip boundary conditions, and the third boundary condition $(9.4 \mathrm{c})$ on the tangential stress has a natural physical interpretation, unlike the alternatives involving higher moments $[5,6,75]$. Solving the above linear system determines

$$
\begin{aligned}
& f_{2}=f_{1}+f_{3}+f_{4}+2 f_{7}+2 f_{8}+\Pi_{x x}^{(0)}, \\
& f_{5}=\frac{1}{2}\left(\Pi_{x x}^{(0)}+U_{\text {wall }}\right)-f_{1}-f_{8}, \\
& f_{6}=\frac{1}{2}\left(\Pi_{x x}^{(0)}-U_{\text {wall }}\right)-f_{3}-f_{7},
\end{aligned}
$$

with $\Pi_{x x}^{(0)}=(1 / 3) \rho+\rho U_{\text {wall }}^{2}$, and $U_{\text {wall }}=U_{0} \sin (\omega t)$ known. We take $\rho=1$ for simplicity, since $\rho$ remains spatially uniform in a shear flow with $\nabla \cdot \mathbf{u}=0$, rather than determining $\rho=\sum_{\alpha} f_{\alpha}$ self-consistently as part of the algebraic system. These boundary conditions supply the distribution functions at the lowermost lattice points at $y=0$ immediate before the collision step. An equivalent calculation supplies boundary conditions for $f_{4}, f_{7}, f_{8}$ at the uppermost lattice points at $y=1$. Since the whole problem is independent of $x$, it is sufficient to use just one point in the $x$ direction with periodic boundary conditions.

Figure 9.1 shows excellent agreement between the analytical solution (9.1) with coefficients (9.2) and the numerical solution computed using the lattice Boltzmann algorithm from Secs. 4 and 5. The parameters are $\omega=4 \pi, \mu=1 / 30, \lambda=1$, $\Lambda=0.01$, for which the formulas (9.2) give $k \approx 68.31$ and $\kappa \approx 7.01$. The simulation used a lattice of 1024 points and a Mach number $M a=\sqrt{3} / 200 \approx 0.0087$. The plot shows the solution at a time of maximum displacement of the wall, $t=(2 n+1 / 2) \pi / \omega$ for integer $n$. The attenuation scale is substantially affected by $\Lambda$, even though $\omega \Lambda \approx 1 / 8$ for these parameters. The corresponding attenuation scale is $\kappa \approx 2.74$ for a linear Maxwell fluid with $\Lambda=0$.

10. The four-roller mill. A configuration of four rollers is commonly used to create two-dimensional extensional flows in the laboratory $[86,34]$. A convenient numerical analog uses the body force $\mathbf{F}=(2 \sin x \cos y,-2 \cos x \sin y)^{\top}$ to create a pattern of Taylor-Green vortices in the doubly-periodic domain $0 \leq x, y \leq 2 \pi$ at zero Reynolds number [88]. For incompressible flow with a velocity field written as $\mathbf{u}=\hat{\boldsymbol{z}} \times \nabla \psi$ in terms of a streamfunction $\psi$, the vorticity equation at finite Reynolds number is

$$
\partial_{t} \omega+[\psi, \omega]=\zeta+\nu^{\prime} \nabla^{2} \omega+4 \sin x \sin y,
$$

where $\omega=\nabla^{2} \psi$, and the Jacobian $[\psi, \omega]=\hat{z} \cdot(\nabla \psi \times \nabla \omega)$. We have separated the Newtonian viscous torque $\nu^{\prime} \nabla^{2} \omega$ from the elastic torque $\zeta=\hat{z} \cdot \nabla \times \nabla \cdot \widetilde{\mathrm{T}}$ using the decomposition $\mathrm{T}=\nu^{\prime} \mathrm{E}+\widetilde{\mathrm{T}}$ for a fluid of unit density. The elastic torque evolves according to the linear Maxwell model

$$
\zeta+\lambda \partial_{t} \zeta=\widetilde{\nu} \nabla^{2} \omega
$$

where $\nu^{\prime}$ and $\widetilde{\nu}$ are related to the parameters $\nu, \lambda, \Lambda$ in the Jeffreys model by

$$
\widetilde{\nu}=\nu(1-\Lambda / \lambda), \quad \nu^{\prime}=\nu \Lambda / \lambda .
$$

The solution to the coupled PDEs (10.1) and (10.2) may be written as

$$
\omega=\hat{\omega}(t) \sin x \sin y, \quad \zeta=\hat{\zeta}(t) \sin x \sin y,
$$

since the forcing term $\sin x \sin y$ is an eigenfunction of the Laplacian, so $\left[\nabla^{-2} \omega, \omega\right]=0$. The two functions $\hat{\omega}(t)$ and $\hat{\zeta}(t)$ evolve according to the coupled ordinary differential equations

$$
d_{t} \hat{\omega}=\hat{\zeta}-2 \nu^{\prime} \hat{\omega}+4, \quad \hat{\zeta}+\lambda d_{t} \hat{\zeta}=-2 \widetilde{\nu} \hat{\omega},
$$




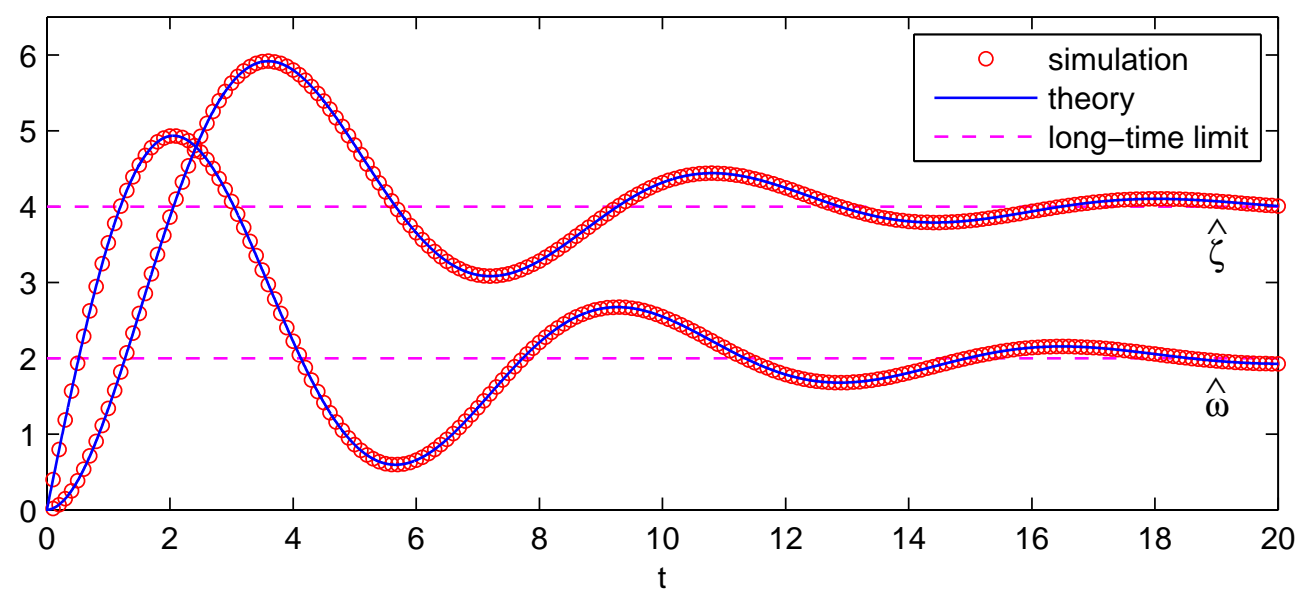

FIG. 10.1. Evolution of the maximum vorticity $|\hat{\omega}|$ and maximum total torque $\left|\hat{\zeta}_{\text {tot }}\right|$ for the doubly-periodic four-roller mill starting from rest with $\nu=1$, $\lambda=2.5$, and $\Lambda=0.01$. Both quantities show an oscillatory approach to their long-time limits $\hat{\omega}_{\infty}=2 / \nu$ and $\left|\hat{\zeta}_{\text {tot }} \infty\right|=4$.
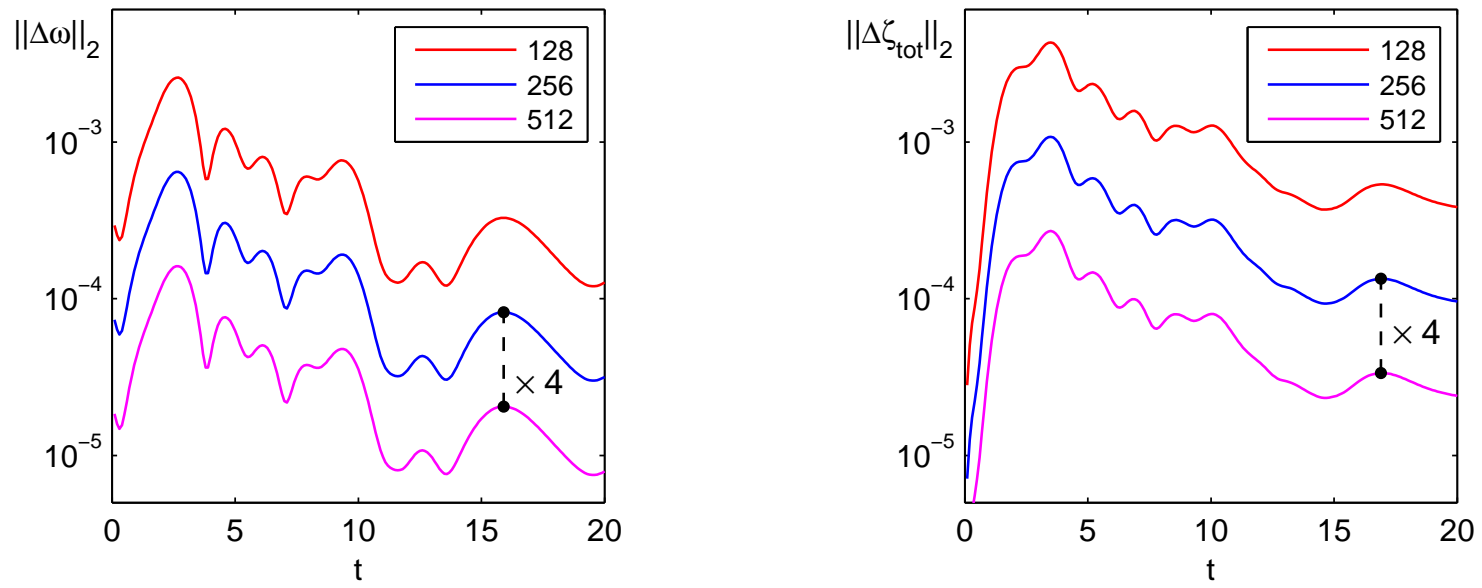

FIG. 10.2. $\ell_{2}$-norm errors in the vorticity field $\omega$ and total torque field $\zeta_{\text {tot }}$ computed on square $N \times N$ grids for $N \in\{128,256,512\}$ (top to bottom). Each simulation was run with a Mach number Ma $=\sqrt{3} \times 1.28 / N$. Doubling $N$ reduces the errors in both $\omega$ and $\zeta_{\text {tot }}$ by a factor of 4 .

whose solutions asymptote to the limiting values $\hat{\omega}_{\infty}=2 / \nu$ and $\hat{\zeta}_{\infty}=4(\Lambda / \lambda-1)$ at long times. The availability of an analytical solution to these equations makes the doubly-periodic four-roller mill a useful benchmark, though the behavior of the Jeffreys fluid is much simpler than the behavior of the Oldroyd-B fluid with its nonlinear stretching terms [88].

Figure 10.1 shows the evolution of the maximum vorticity and maximum total torque for a numerical simulation starting from rest, as compared with the analytical solution of (10.5) with initial conditions $\hat{\omega}(0)=0$ and $\hat{\zeta}(0)=0$. We compare the total torque because the the lattice Boltzmann algorithm computes the total viscoelastic stress $T$, rather than the separate viscous and elastic stresses in the decomposition under (10.1). The corresponding total torque in the analytical solution above is $\zeta_{\text {tot }}=\zeta+\nu^{\prime} \nabla^{2} \omega$ with amplitude $\hat{\zeta}_{\text {tot }}=\hat{\zeta}-2 \nu^{\prime} \hat{\omega}$. The numerical vorticity $\omega=\hat{\boldsymbol{z}} \cdot \nabla \times \mathbf{u}$ and total torque $\zeta_{\text {tot }}=$ $\hat{\boldsymbol{z}} \cdot \nabla \times \nabla \cdot \mathbf{T}=\left(\partial_{x x}-\partial_{y y}\right) T_{x y}+\partial_{x y}\left(T_{y y}-T_{x x}\right)$ were computed by inverting the transformations (8.10) and (6.6) that define $\overline{\mathbf{u}}, \overline{\mathrm{T}}, \overline{\mathrm{M}}$, then spectrally differentiating the components of $\mathbf{u}$ and $\mathrm{T}$ on lattice points. The torque involves only the traceless part of $\mathrm{T}$. The initial conditions correspond to $\mathbf{u}=0, \mathrm{~T}=0$ and $\mathrm{M}=0$ in the lattice Boltzmann formulation, which transform into $\overline{\mathbf{u}}=-\frac{1}{2} \rho^{-1} \Delta t \mathbf{F}, \overline{\mathbf{T}}=0$ and $\overline{\mathbf{M}}=0$ under (8.10) and (6.6).

Figure 10.2 shows the $\ell_{2}$-norm errors in the vorticity and total torque fields relative to the analytical solution $\omega(x, y, t)=$ $\hat{\omega}(t) \sin x \sin y$ and $\zeta_{\text {tot }}(x, y, t)=\hat{\zeta}_{\text {tot }} \sin x \sin y$ for simulations on $N \times N$ lattices with $N \in\{128,256,512\}$. Each simulation was run with a Mach number $M a=\sqrt{3} \times 1.28 / N$. This so-called diffusive scaling [80, 47, 53] balances the $O\left(M a^{2}\right)$ compressibility error with the $O\left(N^{-2}\right)$ spatial truncation errror to give second-order convergence towards the analytical solution of the incompressible fluid equations, as shown in the figure.

11. Three dimensional Arnold-Beltrami-Childress flows. The four-roller mill flow in a linear Jeffreys fluid has an analytical solution because the forcing term $4 \sin x \sin y$ in the vorticity equation is an eigenfunction of the Laplacian. This sets the nonlinear term $\left[\nabla^{-2} \omega, \omega\right]$ to zero. The three-dimensional Arnold-Beltrami-Childress (ABC) flows $[3,42,16]$

$$
\mathbf{u}_{\mathrm{ABC}}=A(0, \sin x, \cos x)+B(\cos y, 0, \sin y)+C(\sin z, \cos z, 0)
$$

in the triply-periodic domain $0 \leq x, y, z<2 \pi$ have the equivalent property of being eigenfunctions of the curl operator $\left(\nabla \times \mathbf{u}_{\mathrm{ABC}}=\mathbf{u}_{\mathrm{ABC}}\right)$. This eliminates the nonlinear $(\nabla \times \mathbf{u}) \times \mathbf{u}$ term in the three-dimensional incompressible Navier-Stokes 


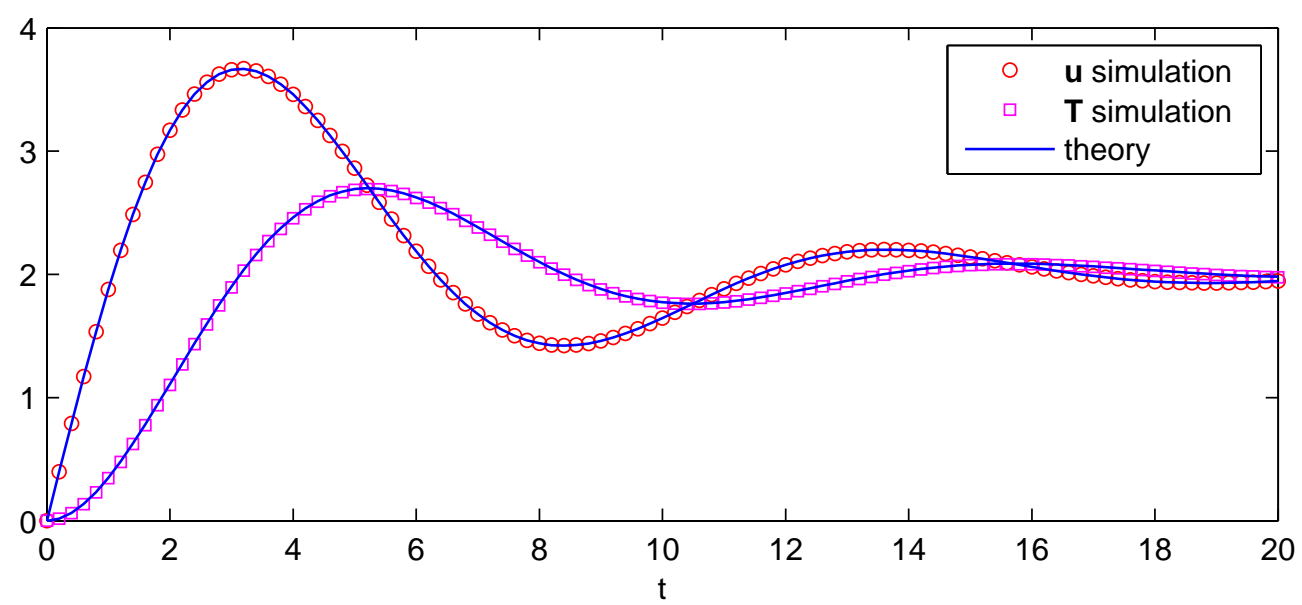

FIG. 11.1. Evolution of the maxima of the velocity vector $\mathbf{u}$ and the total stress component $T_{x y}$ for the ABC flow with $A=B=C=1$ starting from rest with $\nu=1, \lambda=2.5$, and $\Lambda=0.01$.

equations for a fluid of unit density written as

$$
\partial_{t} \mathbf{u}+(\nabla \times \mathbf{u}) \times \mathbf{u}+\nabla\left(p+\frac{1}{2}|\mathbf{u}|^{2}\right)=\nu \nabla \cdot \mathrm{E}
$$

The evolution equations for a linear Jeffreys fluid driven by a body force $\mathbf{F}$ equal to $\mathbf{u}_{\mathrm{ABC}}$ thus reduce to

$$
\partial_{t} \boldsymbol{\omega}=\boldsymbol{\zeta}+\nu^{\prime} \nabla^{2} \boldsymbol{\omega}+\mathbf{F}, \quad \boldsymbol{\zeta}+\lambda \partial_{t} \boldsymbol{\zeta}=\widetilde{\nu} \nabla^{2} \boldsymbol{\omega}
$$

for the vorticity vector $\boldsymbol{\omega}=\nabla \times \mathbf{u}$ and total torque vector $\zeta=\nabla \times \nabla \cdot \mathrm{T}$. These in turn reduce to a pair of ODEs analogous to (10.5) for the amplitudes of $\omega$ and $\zeta$,

$$
d_{t} \hat{\omega}=\hat{\zeta}-\nu^{\prime} \hat{\omega}+1, \quad \hat{\zeta}+\lambda d_{t} \hat{\zeta}=-\widetilde{\nu} \hat{\omega}
$$

The previous factors of 2 are absent because the ABC vector fields are eigenfunctions of the Laplacian with eigenvalue -1 , while $\sin x \sin y$ is an eigenfunction with eigenvalue -2 .

The strain rate tensor $E$ for the velocity field $\mathbf{u}_{\mathrm{ABC}}$ has non-zero components

$$
E_{x y}=B \cos x-C \sin y, \quad E_{x z}=A \cos z-B \sin x, \quad E_{y z}=C \cos y-A \sin z,
$$

and their symmetric pairs. All diagonal components zero. The $x y$ component of the total stress tensor is thus

$$
T_{x y}=(-\hat{\zeta}+\hat{\omega} \Lambda / \lambda)(B \cos x-C \sin y),
$$

and similarly for $T_{x z}$ and $T_{y z}$. Figure 11.1 shows the evolution of the maxima of the velocity vector $\mathbf{u}$ and the total stress component $T_{x y}$ in comparison with the analytical solution (11.6), and the corresponding expression $\mathbf{u}=\hat{\omega} \mathbf{u}_{\mathrm{ABC}}$ for the velocity field. This simulation was run on a grid of $128^{3}$ points using the D3Q27 velocity space lattice [41] at Mach number $M a=\sqrt{3} / 200$.

Figure 11.2 shows the $\ell_{2}$ norms of the differences between the analytical solution $\mathbf{u}=\hat{\omega} \mathbf{u}_{\mathrm{ABC}}$ and the computed velocity field for simulations of $\mathrm{ABC}$ flow with $A=B=C=1$ on $N^{3}$ lattices with $N \in\{32,64,128\}$. As before, each simulation was run with a Mach number $M a=\sqrt{3} \times 0.64 / N$ to balance the compressibility error with the spatial truncation error. Figure 11.2 also shows the second-order convergence in the $\ell_{2}$ norm of the three non-zero components $T_{x y}, T_{x z}, T_{y z}$ of the total stress towards the analytical solution (11.6) and its cyclic permutations

12. Simulations at larger Reynolds numbers. The previous numerical experiments used large viscosity values $(\nu=1)$ to emphasise viscoelastic effects. However, the numerical algorithm is not limited to these viscously-dominated, low Reynolds number flows. Figure 12.1 shows the vertical velocity components at $t=0.5$ for simulations of a modified Taylor-Green vortex evolving from the initial conditions $[87,70]$

$$
u_{x}=\cos 2 \pi x \sin 2 \pi y \cos (2 \pi z+\pi / 4), \quad u_{y}=-\sin 2 \pi x \cos 2 \pi y \cos (2 \pi z+\pi / 4), \quad u_{z}=0
$$

in the triply-periodic domain $0 \leq x, y, z<1$ discretised using a $128^{3}$ lattice with $\nu=10^{-3}$ and $M a=\sqrt{3} / 100$. One simulation shows a Newtonian fluid, and the other shows a Jeffreys fluid with $\lambda=10$ and $\Lambda=10^{-3}$. The differences are small because the viscous and viscoelastic stresses are both small for large Reynolds numbers. 

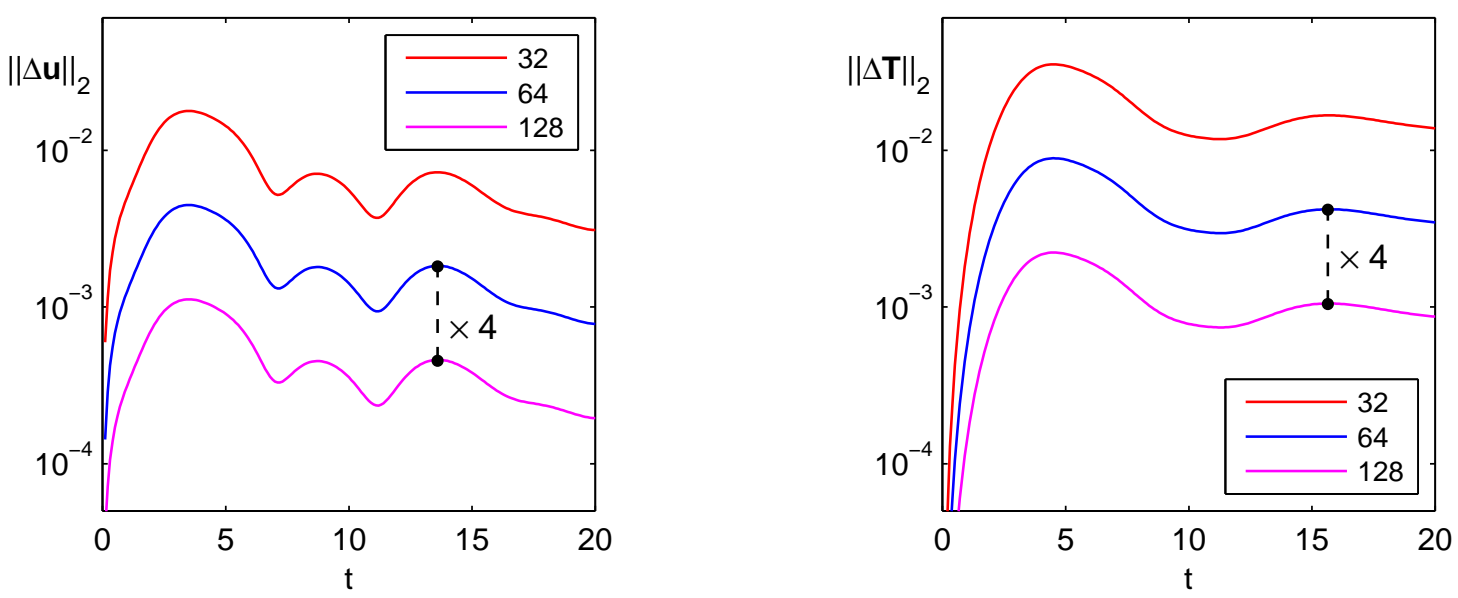

FIG. 11.2. $\ell_{2}$-norm errors in the velocity field $\mathbf{u}$ and the off-diagonal components of the total stress tensor $\boldsymbol{\top}$ from simulations on grids with $N^{3}$ points for $N \in\{32,64,128\}$ (top to bottom). Each simulation was run with a Mach number Ma $=\sqrt{3} \times 0.64 / N$. Doubling $N$ reduces the errors in both $\mathbf{u}$ and $\mathrm{T}$ by a factor of 4 .
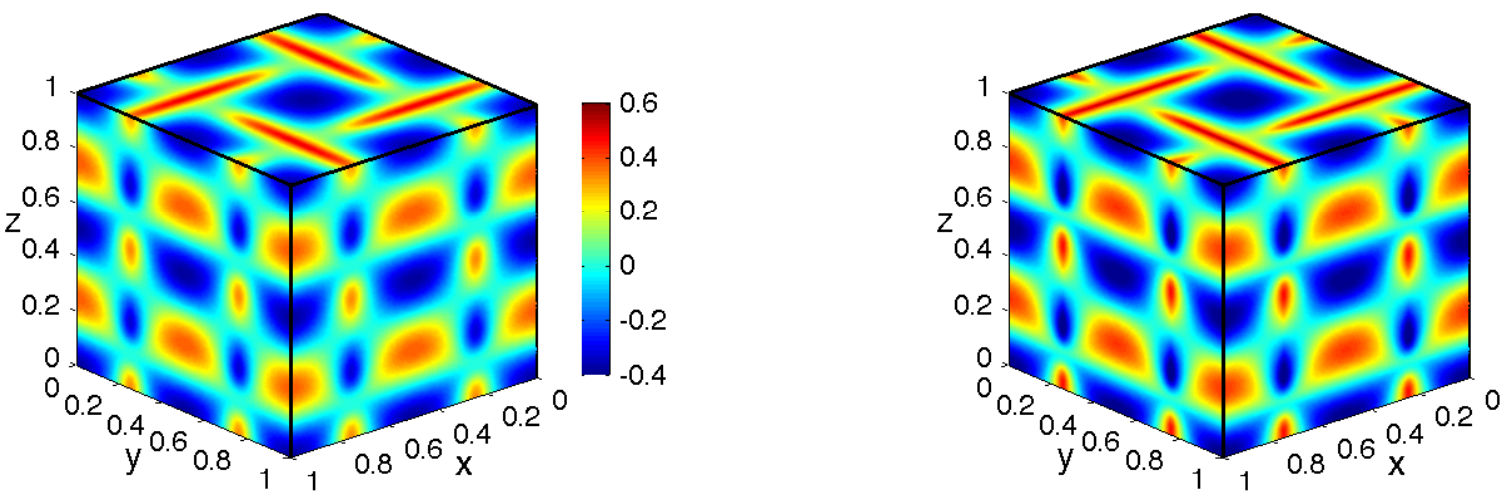

FIG. 12.1. The vertical velocity component at $t=0.5$ for simulations of a modified Taylor-Green vortex with viscosity $\nu=10^{-3}$ for (left) a Newtonian fluid, and (right) a Jeffreys fluid with $\lambda=10$ and $\Lambda=10^{-3}$. Both plots use the same color scale. Differences are small because the deviatoric stresses are small in both flows.

13. Conclusion. In kinetic theory every hydrodynamic quantity must obey the evolution equation implied by the underlying evolution equation for the distribution function. The evolution equation for the pressure tensor given by Maxwell's equation of transfer closely resembles the Oldroyd-B model for viscoelastic liquids. However, the nonlinear coupling between the pressure tensor $\mathrm{P}$ and the velocity gradient $\nabla \mathbf{u}$ in kinetic theory creates a non-objective time derivative that is considered unsuitable for modelling viscoelastic liquids. Moreover, kinetic theory imposes a fixed relation between the stress relaxation time $\tau$ and the dynamic viscosity $\mu=\tau \rho \theta$ for a fluid with density $\rho$ and temperature $\theta$ in energy units. These two coefficients are independent parameters for viscoelastic fluids.

The introduction of a second stress tensor that couples to the first through collisions allows independent adjustment of the two coefficients $\tau$ and $\mu$, while retaining the linear, constant-coefficient property of the discrete Boltzmann equation that allows an efficient and non-dissipative space/time discretisation. Our coupling of the discrete Boltzmann PDE to an abstract second stress, followed by a discretisation using second-order Strang splitting, offers a much simpler alternative to the previous fully discrete models using extra rest particles [36, 37, 59], and extends easily to three dimensional flows driven by body forces. We recover an incompressible linear Jeffreys fluid in the low Mach number limit, even though our starting point, the discrete Boltzmann equation, implies a non-objective convected derivative for the deviatoric stress intead of a simple partial time derivative. Replacing the partial derivative $\partial_{t} \mathrm{M}$ with a finite difference approximation to an objective convected derivative in (4.2) may allow the simulation of nonlinear viscoelastic fluids.

Acknowledgements. It is a pleasure to thank Alexander Wagner for useful correspondence, and to acknowledge the hospitality of the Summer Study Program in Geophysical Fluid Dynamics at Woods Hole Oceanographic Institution, and of the Beijing Computational Science Research Center, where parts of this work were completed. The computations employed the Advanced Research Computing facilities at the University of Oxford, and the Emerald GPU cluster at the e-Infrastructure South Centre for Innovation. The author's research was supported by an Advanced Research Fellowship from the UK Engineering and Physical Sciences Research Council [grant number EP/E054625/1].

Appendix. Modified discrete Boltzmann equation with two parameters.

The standard Boltzmann equation implies the relation $\mu=\tau \rho \theta$ between the dynamic viscosity $\mu$ and the stress relaxation 
time $\tau$. Tsutahara et al. [90] introduced the modified discrete Boltzmann equation

$$
\partial_{t} f_{\alpha}+\boldsymbol{\xi}_{\alpha} \cdot \nabla f_{\alpha}-\frac{\Lambda}{\tau} \boldsymbol{\xi}_{\alpha} \cdot \nabla\left(f_{\alpha}-f_{\alpha}^{(0)}\right)=-\frac{1}{\tau}\left(f_{\alpha}-f_{\alpha}^{(0)}\right)
$$

with an additional term proportional to $\Lambda$ that changes the relation between $\mu$ and $\tau$. The first and second moments of (A.1) give

$$
\begin{array}{r}
\partial_{t}(\rho \mathbf{u})+\nabla \cdot \boldsymbol{\Pi}-\frac{\Lambda}{\tau} \nabla \cdot\left(\boldsymbol{\Pi}-\boldsymbol{\Pi}^{(0)}\right)=0, \\
\partial_{t} \boldsymbol{\Pi}+\nabla \cdot \mathbf{Q}-\frac{\Lambda}{\tau} \nabla \cdot\left(\mathbf{Q}-\mathbf{Q}^{(0)}\right)=-\frac{1}{\tau}\left(\boldsymbol{\Pi}-\boldsymbol{\Pi}^{(0)}\right) .
\end{array}
$$

Putting the approximation $\mathrm{Q}=\mathrm{Q}^{(0)}$ into (A.3) leads to the previous equation (2.13) for $\mathrm{T}$ with relaxation timescale $\tau$. However, the momentum equation (A.2) now becomes

$$
\partial_{t}(\rho \mathbf{u})+\nabla \cdot\left[\mathbf{\Pi}^{(0)}-(1-\Lambda / \tau) \top\right]=0
$$

so the fluid viscosity is $\mu=\rho \theta(\tau-\Lambda)$. The coefficient $\Lambda$ allows the viscosity to be decreased below the value $\mu_{0}=\tau \rho \theta$ previously set by the stress relaxation time $\tau$. However, the left hand side of (A.1) cannot be written as a total time derivative along a straight characteristic, because $\nabla f_{\alpha}^{(0)}$ couples $f_{\alpha}$ to all the $f_{\beta}$ with $\beta \neq \alpha$ through $\rho$ and $\mathbf{u}$. The standard lattice Boltzmann space/time discretisation thus cannot be applied to (A.1). Instead, Tsutahara et al. [90] combined third-order upwind finite differences for spatial derivatives with an explicit second-order Runge-Kutta integration in time. Taking $\Lambda \approx \tau$ enables the viscosity to be reduced without the Runge-Kutta stability condition $\Delta t<2 \tau$ imposing an excessively short restriction on the timestep $\Delta t$.

\section{REFERENCES}

[1] E. Aharonov And D. H. Rothman, Non-Newtonian flow through porous media - a lattice Boltzmann method, Geophys. Res. Lett., 20 (1993), pp. 679-682.

[2] C. K. Aidun And J. R. Clausen, Lattice-Boltzmann method for complex flows, Annu. Rev. Fluid Mech., 42 (2010), pp. 439-472.

[3] V. ARnold, Sur la topologie des écoulements stationnaires des fluides parfaits, C. R. Acad. Sci. Paris, 261 (1965), pp. 17-20.

[4] G. K. BAtchelor, An Introduction to Fluid Dynamics, Cambridge University Press, Cambridge, 1967.

[5] S. BennetT, A lattice Boltzmann model for diffusion of binary gas mixtures, PhD thesis, University of Cambridge, $2010 . \quad$ Available from http://www.dspace.cam.ac.uk/handle/1810/226851

[6] S. Bennett, P. ASInARI, AND P. J. Dellar, A lattice Boltzmann model for diffusion of binary gas mixtures that includes diffusion slip, Int. J. Numer. Meth. Fluids, 69 (2012), pp. 171-189.

[7] P. L. Bhatnagar, E. P. GROss, AND M. KROOK, A model for collision processes in gases. $i$. small amplitude processes in charged and neutral one-component systems, Phys. Rev., 94 (1954), pp. 511-525.

[8] R. B. Bird, R. C. Armstrong, And O. Hassager, Dynamics of Polymeric Liquids, vol. 1, Wiley, New York; Chichester, 1977.

[9] R. B. Bird, O. Hassager, R. C. Armstrong, And C. F. Curtiss, Dynamics of Polymeric Liquids, vol. 2, Wiley, New York; Chichester, 1977.

[10] P. Biscari, C. Cercignani, And M. Slemrod, Time-derivatives and frame-invariance beyond Newtonian fluids, C. R. Acad. Sci. Paris, Ser. IIb, 328 (2000), pp. 417-422.

[11] D. Boger, A highly elastic constant-viscosity fluid, J. Non-Newt. Fluid Mech., 3 (1977), pp. 87-91.

[12] M. E. CATES, O. HENRICH, D. MARENDUZZO, AND K. STRATFORD, Lattice Boltzmann simulations of liquid crystalline fluids: active gels and blue phases, Soft Matter, 5 (2009), pp. 3791-3800.

[13] C. Cercignani, The Boltzmann Equation and its Applications, Springer, New York, 1988.

[14] M. CHAI AND Y. YEOW, Modelling of Boger fluid jets using the Oldroyd-B equation - A comparison of experimental and numerical results, J. NonNewt. Fluid Mech., 29 (1988), pp. 433-442.

[15] S. Chapman and T. G. Cowling, The Mathematical Theory of Non-Uniform Gases, Cambridge University Press, Cambridge, 3rd ed., 1970.

[16] S. ChILDRESS, New solutions of the kinematic dynamo problem, J. Math. Phys., 11 (1970), pp. 3063-3076.

[17] I. C. Christov, Stokes first problem for some non-Newtonian fluids: Results and mistakes, Mech. Res. Comm., 37 (2010), pp. $717-723$.

[18] P. J. DellaR, Lattice kinetic schemes for magnetohydrodynamics, J. Comput. Phys., 179 (2002), pp. 95-126.

[19] - Incompressible limits of lattice Boltzmann equations using multiple relaxation times, J. Comput. Phys., 190 (2003), pp. 351-370.

[20] - Lattice Boltzmann formulation for Braginskii magnetohydrodynamics, Comput. Fluids, 46 (2011), pp. $201-205$.

[21] - An interpretation and derivation of the lattice Boltzmann method using Strang splitting, Comput. Math. Applic., 65 (2013), pp. 129-141.

[22] - Lattice Boltzmann magnetohydrodynamics with current-dependent resistivity, J. Comput. Phys., 237 (2013), pp. 115-131.

[23] D. D’Humières, Generalized lattice-Boltzmann equations, in Rarefied Gas Dynamics: Theory and Simulations, B. D. Shizgal and D. P. Weaver, eds., vol. 159 of Prog. Astronaut. Aeronaut., Washington, D.C., 1994, AIAA, pp. 450-458.

[24] D. D'Humieres AND P. LALlemand, Lattice-gas with transverse-waves, C. R. Acad. Sci. Paris, Ser. II, 317 (1993), pp. $997-1001$.

[25] B. DüNWEg AND A. J. C. LADD, Lattice Boltzmann simulations of soft matter systems, Adv. Poly. Sci., 221 (2009), pp. 1-78.

[26] B. C. EU, On the corotating frame and evolution equations in kinetic theory, J. Chem. Phys., 82 (1985), pp. 3773-3778.

[27] J. D. FERRY, Viscoelastic Properties of Polymers, Wiley, New York, 3rd ed., 1980.

[28] J. D. FERRY, W. M. SAWYER, AND J. N. ASHWORTH, Behavior of concentrated polymer solutions under periodic stresses, J. Polym. Sci., 2 (1947), pp. 593-611.

[29] X. Frank AND H. Z. LI, Complex flow around a bubble rising in a non-Newtonian fluid, Phys. Rev. E, 71 (2005), p. 036309.

[30] - Negative wake behind a sphere rising in viscoelastic fluids: A lattice Boltzmann investigation, Phys. Rev. E, 74 (2006), p. 056307.

[31] G. N. FRANTZISKONIS, Lattice Boltzmann method for multimode wave propagation in viscoelastic media and in elastic solids, Phys. Rev. E, 83 (2011), p. 066703.

[32] M. Frewer, More clarity on the concept of material frame-indifference in classical continuum mechanics, Acta Mech., 202 (2009), pp. $213-246$.

[33] H. Frohlich AND R. SACK, Theory of the rheological properties of dispersions, Proc. Roy. Soc. Lond. Ser. A, 185 (1946), pp. $415-430$.

[34] G. G. Fuller And L. G. LEAL, Flow birefringence of concentrated polymer solutions in two-dimensional flows, J. Polym. Sci. Polym. Phys. Ed., 19 (1981), pp. 557-587.

[35] L. Garcia-Colin, R. Velasco, And F. URIBE, Beyond the Navier-Stokes equations: Burnett hydrodynamics, Phys. Rep., 465 (2008), pp. 149-189. 
[36] L. Giraud, D. D’Humieres, And P. Lallemand, A lattice-Boltzmann model for visco-elasticity, Int. J. Mod. Phys. C, 8 (1997), pp. $805-815$.

[37] L. GiRAUD, D. D'HumiERES, AND P. LALLEMAND, A lattice Boltzmann model for Jeffreys viscoelastic fluid, Europhys. Lett., 42 (1998), pp. 625-630.

[38] H. GRAD, Note on N-dimensional Hermite polynomials, Comm. Pure Appl. Math., 2 (1949), pp. 325-330.

[39] E. Hairer, C. Lubich, AND G. WANner, Geometric Numerical Integration: Structure-Preserving Algorithms for Ordinary Differential Equations, Springer, 2nd ed., 2006.

[40] X. HE, S. Chen, And G. D. Doolen, A novel thermal model of the lattice Boltzmann method in incompressible limit, J. Comput. Phys., 146 (1998), pp. 282-300.

[41] X. HE AND L.-S. Luo, Theory of the lattice Boltzmann method: From the Boltzmann equation to the lattice Boltzmann equation, Phys. Rev. E, 56 (1997), pp. 6811-6817.

[42] M. HÉnon, Sur la topologie des lignes de courant dans un cas particulier, C. R. Acad. Sci. Paris, 262 (1966), pp. $312-314$.

[43] M. HÉnon, Viscosity of a lattice gas, Complex Sys., 1 (1987), pp. 763-789.

[44] F. J. Higuera, S. SUCCI, AND R. BENZI, Lattice gas dynamics with enhanced collisions, Europhys. Lett., 9 (1989), pp. $345-349$.

[45] S. Hou, J. D. Sterling, C. Chen, And G. D. Doolen, A lattice Boltzmann subgrid model for high Reynolds number flows, in Pattern Formation and Lattice Gas Automata, A. T. Lawniczak and R. Kapral, eds., vol. 6 of Fields Institute Proceedings, 1996, pp. 151-166.

[46] E. IKENBERRY AND C. TRuesdell, On the pressures and the flux of energy in a gas according to Maxwell's kinetic theory, I, J. Ratl. Mech. Anal., 5 (1956), pp. 1-54.

[47] T. InAmuro, M. Yoshino, AND F. OGino, Accuracy of the lattice Boltzmann method for small Knudsen number with finite Reynolds number, Phys. Fluids, 9 (1997), pp. 3535-3542.

[48] I. IsPolatov AND M. GRANT, Lattice Boltzmann method for viscoelastic fluids, Phys. Rev. E, 65 (2002), p. 056704.

[49] D. F. JAMES, Boger fluids, Annu. Rev. Fluid Mech., 41 (2008), pp. 129-142.

[50] H. JEFFREYS, The Earth, Cambridge University Press, 1929.

[51] D. D. JOSEPH, Fluid Dynamics of Viscoelastic Liquids, Springer, Berlin, 1990.

[52] D. D. Joseph And L. Preziosi, Addendum to the paper “heat waves”, Rev. Mod. Phys., 62 (1990), pp. $375-391$.

[53] M. JUnK, A. KLAR, AND L.-S. LuO, Asymptotic analysis of the lattice Boltzmann equation, J. Comput. Phys., 210 (2005), pp. 676-704.

[54] S. KARRA, Modeling electrospinning process and a numerical scheme using lattice Boltzmann method to simulate viscoelastic fluid flows, Master's thesis, Texas A\&M University, 2007.

[55] J. M. V. A. Koelman, A simple lattice Boltzmann scheme for Navier-Stokes fluid flow, Europhys. Lett., 15 (1991), pp. $603-607$.

[56] A. L. KUPERSHTOKH, New method of incorporating a body force term into the lattice Boltzmann equation, in Proc. 5th International EHD Workshop, August 30-31, 2004, Poitiers, France, 2004, pp. 241-246.

[57] - Criterion of numerical instability of liquid state in LBE simulations, Comput. Math. Applic., 59 (2010), pp. $2236-2245$.

[58] A. J. C. LADD, Numerical simulations of particulate suspensions via a discretized Boltzmann equation. Part 1. Theoretical foundation, J. Fluid Mech., 271 (1994), pp. 285-309.

[59] P. Lallemand, D. D'Humieres, L.-S. Luo, AND R. RuBinstein, Theory of the lattice Boltzmann method: Three-dimensional model for linear viscoelastic fluids, Phys. Rev. E, 67 (2003), pp. 021203-19.

[60] P. Lallemand AND L.-S. LuO, Theory of the lattice Boltzmann method: Dispersion, dissipation, isotropy, Galilean invariance, and stability, Phys. Rev. E, 61 (2000), pp. 6546-6562.

[61] L. D. LANDAu AND E. M. Lifshitz, Fluid Mechanics, Pergamon, Oxford, 2nd ed., 1987.

[62] H.-B. Li AND H.-P. FANG, Lattice Boltzmann simulation of transverse wave travelling in Maxwell viscoelastic fluid, Chinese Phys., 13 (2004), pp. 2087-2090.

[63] O. MAlaspinas, N. FiÉTIER, AND M. DEville, Lattice Boltzmann method for the simulation of viscoelastic fluid flows, J. Non-Newt. Fluid Mech., 165 (2010), pp. 1637-1653.

[64] G. MARRUCCI, The free energy constitutive equation for polymer solutions from the dumbbell model, Trans. Soc. Rheol., 16 (1972), pp. $321-330$.

[65] J. C. Maxwell, On the dynamical theory of gases, Phil. Trans. Roy. Soc. Lond., 157 (1867), pp. 49-88.

[66] G. R. McNamara, A. L. GarCiA, AND B. J. Alder, Stabilization of thermal lattice Boltzmann models, J. Statist. Phys., 81 (1995), pp. $395-408$.

[67] J. G. OLDROYD, On the formulation of rheological equations of state, Proc. R. Soc. Lond. A, 200 (1950), pp. $523-541$.

[68] J. ONISHI, Y. CHEN, AND H. OHASHI, A lattice Boltzmann model for polymeric liquids, Progress Computational Fluid Dynamics, 5 (2005), pp. $75-84$.

[69] - Dynamic simulation of multi-component viscoelastic fluids using the lattice Boltzmann method, Physica A, 362 (2006), pp. 84-92.

[70] S. A. ORSZAG, Numerical simulation of the Taylor-Green vortex, Lect. Notes Comput. Sci., 11 (1974), pp. 50-64.

[71] P. Oswald, Rheophysics, Cambridge University Press, Cambridge, 2009.

[72] T. N. Phillips AND G. W. RoberTs, Lattice Boltzmann models for non-Newtonian flows, IMA J. Appl. Math., 76 (2011), pp. $790-816$.

[73] G. Prilutski, R. Gupta, T. SRIDhar, AND M. RYAN, Model viscoelastic liquids, J. Non-Newt. Fluid Mech., 12 (1983), pp. $233-241$.

[74] Y. H. QIAN, D. D'HumièReS, AND P. LALLEMAND, Lattice BGK models for the Navier-Stokes equation, Europhys. Lett., 17 (1992), pp. 479-484.

[75] T. REIS AND P. J. DELlAR, Lattice Boltzmann simulations of pressure-driven flows in microchannels using Navier-Maxwell slip boundary conditions, Phys. Fluids, 24 (2012), pp. 112001-18.

[76] M. RENARDY, Mathematical Analysis of Viscoelastic Flows, SIAM, Philadelphia, 2000.

[77] D. RisSo AND P. CORDERO, Generalized hydrodynamics for a Poiseuille flow: Theory and simulations, Phys. Rev. E, 58 (1998), pp. $546-553$.

[78] J. SMAGORInSKy, General circulation experiments with the primitive equations, Mon. Wea. Rev., 91 (1963), pp. 99-164.

[79] J. A. SOMERS, Direct simulation of fluid flow with cellular automata and the lattice-Boltzmann equation, Appl. Sci. Res., 51 (1993), pp. 127-133.

[80] Y. Sone, Kinetic Theory and Fluid Dynamics, Birkhäuser, Boston, 2002.

[81] J. D. STERling And S. Chen, Stability analysis of lattice Boltzmann methods, J. Comput. Phys., 123 (1996), pp. 196-206.

[82] M. Stiebler, M. KrafCZyK, S. Freudiger, AND M. Geier, Lattice Boltzmann large eddy simulation of subcritical flows around a sphere on non-uniform grids, Comput. Math. Applic., 61 (2011), pp. 3475-3484.

[83] G. G. STOKES, On the effect of the internal friction of fluids on the motion of pendulums, Cambridge Philos. Trans., 9 (1851), pp. 8-106.

[84] G. STRANG, On the construction and comparison of difference schemes, SIAM J. Numer. Anal., 5 (1968), pp. $506-517$.

[85] R. I. TANNER, Note on the Rayleigh problem for a visco-elastic fluid, Z. Angew. Math. Phys., 13 (1962), pp. 573-580.

[86] G. I. TAYLOR, The formation of emulsions in definable fields of flow, Proc. Roy. Soc. Lond. Ser. A, 146 (1934), pp. 501-523.

[87] G. I. TAYlor AND A. E. GREEN, Mechanism of the production of small eddies from large ones, Proc. Roy. Soc. Lond. Ser. A, 158 (1937), pp. 499-521.

[88] B. Thomases AND M. Shelley, Emergence of singular structures in Oldroyd-B fluids, Phys. Fluids, 19 (2007), p. 103103.

[89] M. Tij, M. SABbane, AND A. Santos, Nonlinear Poiseuille flow in a gas, Phys. Fluids, 10 (1998), pp. $1021-1027$.

[90] M. TsuTAhARA, T. KATAOKA, K. SHIKATA, AND N. TAKADA, New model and scheme for compressible fluids of the finite difference lattice Boltzmann method and direct simulations of aerodynamic sound, Comput. Fluids, 37 (2008), pp. 79-89.

[91] F. J. Uribe AND A. L. GARCIA, Burnett description for plane Poiseuille flow, Phys. Rev. E, 60 (1999), pp. $4063-4078$.

[92] A. J. WAGNER, Derivation of a non-objective Oldroyd model from the Boltzmann equation. arXiv cond-mat/0105067, 2001.

[93] A. J. Wagner AND J. M. Yeomans, Phase separation under shear in two-dimensional binary fluids, Phys. Rev. E, 59 (1999), pp. $4366-4373$.

[94] L. C. Woods, Frame-indifferent kinetic theory, J. Fluid Mech., 136 (1983), pp. 423-433.

[95] H. YU, L.-S. LUO, AND S. S. GiRIMAJ, LES of turbulent square jet flow using an MRT lattice Boltzmann model, Comput. Fluids, 35 (2006), pp. 957-965.

[96] R. ZWANZIG, Nonlinear shear viscosity of a gas, J. Chem. Phys., 71 (1979), pp. 4416-4420. 